\title{
Back reaction of 4D conformal fields on static black-hole geometry
}

\author{
Pei-Ming Ho, ${ }^{a}$ Hikaru Kawai, ${ }^{b}$ Yoshinori Matsuo $^{a}$ and Yuki Yokokura ${ }^{c}$ \\ ${ }^{a}$ Department of Physics and Center for Theoretical Physics, \\ National Taiwan University, Taipei 106, Taiwan, R.O.C. \\ ${ }^{b}$ Department of Physics, Kyoto University, \\ Kitashirakawa, Kyoto 606-8502, Japan \\ ${ }^{c}$ iTHEMS Program, RIKEN, \\ Wako, Saitama 351-0198, Japan \\ E-mail: pmho@phys.ntu.edu.tw, hkawai@gauge.scphys.kyoto-u.ac.jp, \\ matsuo@phys.ntu.edu.tw, yuki.yokokura@riken.jp
}

ABSTRACT: Static, spherically symmetric black hole solutions to the semi-classical Einstein equation are studied, including the effect of the quantum energy-momentum tensor for conformal matters with 4D Weyl anomaly. Through both perturbative and non-perturbative methods, we show that the quantum effect can play a crucial role in shaping the nearhorizon geometry, and that the existence of the horizon requires fine-tuning.

Keywords: Black Holes, Classical Theories of Gravity

ARXIV EPRINT: 1807.11352 


\section{Contents}

1 Introduction 2

2 Semi-classical einstein equation and 4D conformal matter 3

2.1 Semi-classical Einstein equation 3

2.2 Energy-momentum tensor and Weyl anomaly 5

3 General perturbative solution in areal radius $\quad 8$

3.1 Perturbative analysis 8

$\begin{array}{ll}3.1 .1 & \text { Analysis in the limit } r \rightarrow \infty\end{array}$

$\begin{array}{ll}\text { 3.1.2 Analysis in the limit } r \rightarrow a & 11\end{array}$

$\begin{array}{lll}3.2 & \text { Classification of solutions } & 12\end{array}$

3.2.1 Wormhole-like throat $(q<0) \quad 13$

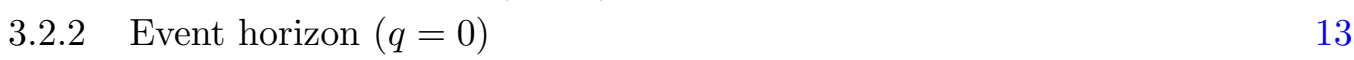

$\begin{array}{lll}3.2 .3 & \text { No neck \& no horizon }(q>0) & 14\end{array}$

$\begin{array}{lll}3.3 & \text { Higher order corrections } & 14\end{array}$

$\begin{array}{lll}3.4 & \text { Time-dependent perturbations } & 15\end{array}$

4 General perturbative solution in proper radial coordinate $\quad \mathbf{1 5}$

$\begin{array}{ll}4.1 & \text { Wormhole-like throat }(q<0) \\ & 16\end{array}$

$\begin{array}{lll}4.2 & \text { Event horizon }(q=0) & 17\end{array}$

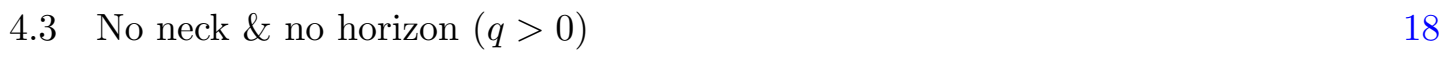

$\begin{array}{lll}4.4 & \text { Higher order corrections } & 18\end{array}$

5 Perturbative solution near Schwarzschild radius $\quad 19$

5.1 Wormhole-like throat $(q<0) \quad 21$

5.2 Event horizon $(q=0) \quad 21$

5.3 No neck \& no horizon $(q>0) \quad 22$

5.4 Relation to expansion around Schwarzschild metric 22

$6 \quad$ Non-perturbative analysis $\quad 23$

6.1 Solving $A(z)$ and $B(z) \quad 24$

6.2 Wormhole-like throat $(q<0) \quad 26$

6.3 Event horizon $(q=0) \quad 28$

6.4 No neck \& no horizon $(q>0) \quad 30$

$\begin{array}{lll}7 & \text { Conclusion } & 30\end{array}$ 


\section{Introduction}

Since Hawking's proposal [1] that a black hole can completely evaporate through Hawking radiation, physicists have realized that quantum effects, despite its weakness, have the potential to affect the large-scale structure of black holes. However, to this day, there has not yet been a satisfactory understanding on this topic, leaving many unsettled issues, including most notably the information loss paradox [2-5] and related proposals such as the fuzzball [6-10] and the firewall [11-13]. Many believe that a rigorous detailed analysis including the quantum effect is imperative.

Hence we aim to provide a rigorous detailed description of the black-hole geometry with the back reaction of quantum fields taken into consideration. In this paper, we focus on static, spherically symmetric black holes. We will give explicit expressions of general solutions to the semi-classical Einstein equation. Following Christensen and Fulling [14], we assume that the underlying quantum fields responsible for the quantum energy-momentum tensor are 4D conformal matters. Unlike its 2D analogue [15], its energy-momentum tensor is not uniquely fixed by the trace anomaly and conservation law. Instead of making additional assumptions to uniquely determine the energy-momentum tensor, we keep its full generality in our analysis.

There are numerous related works in the literature. Let us comment on some of those closely related. Vacuum energy-momentum tensors derived from 2D models of quantum field theories are extensively studied in ref. [16]. It was shown that, depending on the quantum model of vacuum energy-momentum tensor and the vacuum state, the backreacted near-horizon geometry falls into three qualitatively different classes. In one of the three classes, the back reaction of quantum fields is insignificant, while the event horizon is removed in the other two classes.

In one of the two classes that are horizonless, the horizon is replaced by a local minimum of the areal radius, resembling the throat of a traversable wormhole [16]. Similar results were also obtained in refs. [17, 18]. (The resemblance between the black holes and wormholes was also noted in refs. [19-21] based on different reasonings.) The static geometry of the interior space with a star composed of an incompressible fluid or a thin shell was studied in ref. [22]. The dynamical case including the effect of Hawking radiation was explored via numerical simulation in ref. [23], and then analytically in ref. [24].

In the other horizonless class of solutions, there is neither a horizon nor a wormhole-like structure [16].

The progress achieved in this work is mainly the use of 4D (instead of 2D) models of quantum vacuum energy-momentum, and its generality that covers all static solutions with spherical symmetry. All three classes of solutions found in various $2 \mathrm{D}$ models of vacuum energy are present in this $4 \mathrm{D}$ model, and we note that the presence of the event horizon requires fine tuning. The back reaction due to $4 \mathrm{D}$ conformal fields has also been studied in ref. [25], but it was done in a manner different from this work, and only the wormhole-like class was discussed. Furthermore, we emphasize the mathematical rigor of our results, with our calculations carried out in both perturbative and non-perturbative approaches.

The plan of this paper is as follows. We first lay out in section 2 the assumptions behind the mathematical formulation we use to determine the black-hole geometry. The 
general perturbative solution at the first order is given in section 3, section 4 and section 5 in three different coordinate systems, each with its advantages and disadvantages. The non-perturbative analysis is carried out in section 6 . The result is consistent with the perturbative solution. The perturbative and non-perturbative solutions together depict a comprehensive picture of the black-hole geometry.

\section{Semi-classical einstein equation and 4D conformal matter}

\subsection{Semi-classical Einstein equation}

In this section, we define the theoretical framework on which the analysis in this paper is based. It is essentially Einstein's theory of gravity sourced by 4D conformal quantum fields through its expectation value of the quantum energy-momentum operator.

First, we assume that the space-time geometry is determined by the semi-classical Einstein equation

$$
G_{\mu \nu}=\kappa\left\langle T_{\mu \nu}\right\rangle
$$

at large scales. Here $\left\langle T_{\mu \nu}\right\rangle$ is the expectation value of the quantum energy-momentum operator $T_{\mu \nu}$ in the underlying quantum field theory. A priori $\left\langle T_{\mu \nu}\right\rangle$ does not have to be the vacuum expectation value. But in the perturbative calculation, we will assume that $\left\langle T_{\mu \nu}\right\rangle$ is of $\mathcal{O}\left(\kappa^{0}\right)$, so that the right hand side of eq. (2.1) vanishes in the classical limit $\kappa \rightarrow 0$. In this sense, it is a vacuum expectation value which comes purely from quantum effects. On the other hand, in the non-perturbative analysis, it can be the expectation value of an arbitrary state.

In this work, we further assume that the energy-momentum tensor $\left\langle T_{\mu \nu}\right\rangle$ in eq. (2.1) is given as that of $4 \mathrm{D}$ conformal quantum fields. The advantage of considering conformal matters is that $\left\langle T_{\mu \nu}\right\rangle$ is constrained by the Weyl anomaly, leaving fewer uncertainties in $\left\langle T_{\mu \nu}\right\rangle$, which is typically difficult to evaluate directly.

For $4 \mathrm{D}$ conformal quantum fields, the trace of the energy-momentum tensor is given by the $4 \mathrm{D}$ Weyl anomaly

$$
\left\langle T^{\mu}{ }_{\mu}\right\rangle=c_{4} \mathcal{F}+a_{4} \mathcal{G}
$$

which depends on two conformal charges $c_{4}$ and $a_{4}$ characterizing the conformal fields. Here

$$
\begin{aligned}
\mathcal{F} & \equiv C^{\mu \nu \lambda \rho} C_{\mu \nu \lambda \rho}=R^{\mu \nu \lambda \rho} R_{\mu \nu \lambda \rho}-2 R^{\mu \nu} R_{\mu \nu}+\frac{1}{3} R^{2}, \\
\mathcal{G} & \equiv R^{\mu \nu \lambda \rho} R_{\mu \nu \lambda \rho}-4 R^{\mu \nu} R_{\mu \nu}+R^{2}
\end{aligned}
$$

where $C$ is the Weyl tensor and $\mathcal{G}$ is the Gauss-Bonnet term.

The last assumption we shall make in this paper is that the configurations under study are static and spherically symmetric. Locally, the metric can be put in the form

$$
d s^{2}=-e^{\rho(r)}\left[d t^{2}-\frac{d r^{2}}{F(r)}\right]+r^{2} d \Omega^{2} .
$$


The coordinate $r$ is called the "areal radius", in terms of which the area of a symmetric sphere is always $4 \pi r^{2}$. It is not necessarily monotonically increasing in the radial direction. We also define a "proper radial coordinate" $z$ by

$$
d z^{2}=\frac{e^{\rho(r)}}{F(r)} d r^{2}
$$

The metric can then be expressed as

$$
d s^{2}=-\frac{e^{A(z)}}{B^{2}(z)} d t^{2}+d z^{2}+B(z) d \Omega^{2},
$$

where the two parametric functions $A(z)$ and $B(z)$ are related to $\rho(r)$ and $F(r)$ by eq. (2.6) and

$$
r^{2}=B(z), \quad e^{\rho(r)}=\frac{e^{A(z)}}{B^{2}(z)} .
$$

For a static, spherically symmetric configuration, the only non-vanishing components of the energy-momentum tensor $\left\langle T_{\mu \nu}\right\rangle$ are

$$
\left\langle T^{t}{ }_{t}\right\rangle, \quad\left\langle T_{r}^{r}\right\rangle=\left\langle T_{z}^{z}\right\rangle, \quad\left\langle T^{\theta}{ }_{\theta}\right\rangle=\left\langle T_{\phi}^{\phi}\right\rangle
$$

The three independent components of the energy-momentum tensor (say, $\left\langle T^{t}{ }_{t}\right\rangle,\left\langle T^{r}{ }_{r}\right\rangle$ and $\left.\left\langle T^{\theta}{ }_{\theta}\right\rangle\right)$ are constrained by the conservation law

$$
\nabla_{\mu}\left\langle T^{\mu}{ }_{\nu}\right\rangle=0
$$

as well as the anomaly equation (2.2). There is thus only one independent functional degree of freedom in the energy-momentum tensor. We can arbitrarily specify $\left\langle T^{r}{ }_{r}\right\rangle$ (or $\left\langle T^{z}{ }_{z}\right\rangle$ ) to be any given function of $r$ (or $z$ ) from which all other components of $\left\langle T_{\mu \nu}\right\rangle$ are fixed.

The metric (2.5) (or (2.7)) has two independent functional parameters $\rho(r)$ and $F(r)$ (or $A(z)$ and $B(z)$ ) to be solved from the semi-classical Einstein equation (2.1). Correspondingly, only two of the 10 equations in eq. (2.1) are independent. For conformal matters, it is convenient to take the trace of the semi-classical Einstein equation

$$
G^{\mu}{ }_{\mu}=\kappa\left\langle T^{\mu}{ }_{\mu}\right\rangle
$$

where $\left\langle T^{\mu}{ }_{\mu}\right\rangle$ is given by the Weyl anomaly (2.2), as one of the two independent equations. In the following, we will take the other independent equation to be

$$
\begin{aligned}
& G_{r}^{r}=\kappa\left\langle T^{r}{ }_{r}\right\rangle, \\
& \text { or } \\
& G^{z}{ }_{z}=\kappa\left\langle T^{z}{ }_{z}\right\rangle,
\end{aligned}
$$

depending on our choice of coordinates. 


\subsection{Energy-momentum tensor and Weyl anomaly}

The most general static, spherically symmetric stress tensor constrained by the conservation law has only two functional degrees of freedom. Following Christensen and Fulling [14], the energy-momentum tesnor can be parametrized by the trace $\left\langle T_{\mu}^{\mu}\right\rangle$ and

$$
\Theta \equiv\left\langle T_{\theta}^{\theta}\right\rangle-\frac{1}{4}\left\langle T_{\mu}^{\mu}\right\rangle
$$

In addition, there are 2 dimensionless constant parameters $K$ and $Q$ which are a priori of order 1 , corresponding to different choices of boundary conditions.

For the Schwarzschild background (2.5) with

$$
\rho(r)=\log \left(1-\frac{a}{r}\right), \quad F(r)=\left(1-\frac{a}{r}\right)^{2},
$$

we obtain the following expression for the most general time-independent, conserved and spherically symmetric stress tensor by integrating the conservation law;

$$
\begin{aligned}
\left\langle T^{t}{ }_{t}\right\rangle & =-\frac{1}{r(r-a)}[q+H(r)+G(r)]+\frac{1}{2}\left\langle T_{\mu}^{\mu}(r)\right\rangle-2 \Theta(r), \\
\left\langle T^{r}{ }_{r}\right\rangle & =\frac{1}{r(r-a)}[q+H(r)+G(r)], \\
\left\langle T^{t}{ }_{r}\right\rangle & =\frac{1}{(r-a)^{2}} k, \\
\left\langle T^{\theta}{ }_{\theta}\right\rangle & =\Theta(r)+\frac{1}{4}\left\langle T_{\mu}^{\mu}\right\rangle,
\end{aligned}
$$

while

$$
\begin{aligned}
\left\langle T^{r}{ }_{t}\right\rangle & =-\left(1-\frac{a}{r}\right)^{2}\left\langle T_{r}^{t}{ }_{r}\right\rangle, \\
\left\langle T^{\phi}{ }_{\phi}\right\rangle & =\left\langle T^{\theta}{ }_{\theta}\right\rangle,
\end{aligned}
$$

and the two functions $H$ and $G$ are defined by

$$
\begin{aligned}
& H(r)=\frac{1}{2} \int_{a}^{r}\left(r^{\prime}-\frac{a}{2}\right)\left\langle T_{\mu}^{\mu}\left(r^{\prime}\right)\right\rangle d r^{\prime}, \\
& G(r)=2 \int_{a}^{r}\left(r^{\prime}-\frac{3 a}{2}\right) \Theta\left(r^{\prime}\right) d r^{\prime} .
\end{aligned}
$$

The integration constants $q$ and $k$ are related to the parameters $Q$ and $K$ in ref. [14] via

$$
\begin{aligned}
q & =\frac{4(Q-K)}{a^{2}}, \\
k & =\frac{4 K}{a^{2}},
\end{aligned}
$$

which are of order $\mathcal{O}\left(a^{-2}\right)$.

Here, $Q=0$, or equivalently, $q=-k$ does not lead to the divergent outgoing energy flux but the incoming energy flux diverges at the past horizon, while the condition $q=k$ 
gives divergence of the outgoing energy flux at the future horizon but no divergence in incoming energy flux. The condition $q=k=0$, gives no divergent energy flux while $q=0$ generally provides non-zero energy flux in the asymptotic region. For example, the energymomentum tensor which satisfies the conditions $\left\langle T^{\theta}{ }_{\theta}\right\rangle=0$ and the anomaly condition (2.2) has the following asymptotic behavior;

$$
\begin{gathered}
\left\langle T^{t}{ }_{t}(r \rightarrow \infty)\right\rangle=-\frac{a^{2} q+\frac{6}{5}\left(c_{4}+a_{4}\right)}{a^{2} r^{2}}+\mathcal{O}\left(r^{-3}\right), \\
\left\langle T^{r}{ }_{r}(r \rightarrow \infty)\right\rangle=\frac{a^{2} q+\frac{6}{5}\left(c_{4}+a_{4}\right)}{a^{2} r^{2}}+\mathcal{O}\left(r^{-3}\right) .
\end{gathered}
$$

The condition $q=k=0$ corresponds to the Hartle-Hawking vacuum, in which the incoming and outgoing energy fluxes are finite but balanced with each other. For $q=-\frac{6\left(c_{4}+a_{4}\right)}{5 a^{2}}$ and $k=0$, the incoming and outgoing energy fluxes in the asymptotic region are zero but there is divergence at $r=a$. This condition corresponds to the Boulware vacuum. The Hartle-Hawking vacuum was considered physical, and the Boulware vacuum unphysical, because of the divergence in the energy flux. In this work, we do not jump into the same conclusion before closely examining the solutions.

Note that if $k \neq 0$, the energy-momentum tensor breaks the time-reversal symmetry, so the metric will not be static, even though the energy-momentum tensor is timeindependent. We shall assume that $k=0$ except briefly commentting on the case $k \neq 0$ in section 3.4.

Explicit expressions of the energy-momentum tensor can now be given as follows. For the Schwarzschild background,

$$
R^{\mu \nu \lambda \rho} R_{\mu \nu \lambda \rho}=\frac{12 a^{2}}{r^{6}}, \quad R^{\mu \nu} R_{\mu \nu}=0, \quad R=0 .
$$

As a result,

$$
\mathcal{F}=\mathcal{G}=\frac{12 a^{2}}{r^{6}}
$$

and the trace anomaly $(2.2)$ is

$$
\left\langle T^{\mu}{ }_{\mu}\right\rangle=\frac{12\left(c_{4}+a_{4}\right) a^{2}}{r^{6}} .
$$

At the first order in the perturbation theory, this is the only place where the conformal charges appear in the semi-classical Einstein equation, hence they only appear in the combination $\left(c_{4}+a_{4}\right)$ in the first order perturbative solution.

For the Weyl anomaly (2.30), we have

$$
\begin{aligned}
& H(r)=\frac{3\left(c_{4}+a_{4}\right)\left(3 r^{5}-5 a^{4} r+2 a^{5}\right)}{10 a^{2} r^{5}} \\
& G(r)=\frac{3\left(c_{4}+a_{4}\right)\left(r^{5}+5 a^{4} r-6 a^{5}\right)}{10 a^{2} r^{5}}+2 \int_{a}^{r}\left(r^{\prime}-\frac{3 a}{2}\right)\left\langle T^{\theta}{ }_{\theta}\left(r^{\prime}\right)\right\rangle d r^{\prime} .
\end{aligned}
$$

One can thus compute the energy-momentum tensor using eqs. (2.16)-(2.19) for the Schwarzschild background. 
The expressions above given in terms of the areal radius $r$ can be easily generalized to an arbitrary radial coordinate $y$ for the most general static, spherically symmetric background,

$$
d s^{2}=-e^{\rho(y)} d t^{2}+h(y) d y^{2}+r^{2}(y) d \Omega^{2} .
$$

The radial component of the metric $h(y)$ is related to those in other coordinates as

$$
h(y) d y^{2}=\frac{e^{\rho(r)}}{F(r)} d r^{2}=d z^{2} .
$$

For instance, $h(y)=1$ for $y=z$, and $h(y)=\frac{e^{\rho(r)}}{F(r)}$ for $y=r$. By integrating the conservation equations, the energy-momentum tensor is found to be

$$
\begin{aligned}
\left\langle T^{t}{ }_{t}\right\rangle & =-\frac{e^{-\rho(y)}}{r^{2}(y)}[q+H(y)+G(y)]+\frac{1}{2}\left\langle T_{\mu}^{\mu}(y)\right\rangle-2 \Theta(y), \\
\left\langle T^{y}{ }_{y}\right\rangle & =\frac{e^{-\rho(y)}}{r^{2}(y)}[q+H(y)+G(y)], \\
\left\langle T_{t y}\right\rangle & =-\frac{k e^{-\rho(y) / 2} \sqrt{h(y)}}{r^{2}(y)}, \\
\left\langle T^{\theta}{ }_{\theta}\right\rangle & =\left\langle T^{\phi}{ }_{\phi}\right\rangle=\Theta(y)+\frac{1}{4}\left\langle T_{\mu}^{\mu}\right\rangle,
\end{aligned}
$$

where $H$ and $G$ are given by

$$
\begin{aligned}
& H(y)=\frac{1}{4} G(y)+\frac{1}{2} \int_{\bar{y}}^{y} e^{\rho\left(y^{\prime}\right)} \rho^{\prime}\left(y^{\prime}\right) r^{2}\left(y^{\prime}\right)\left\langle T_{\mu}^{\mu}\left(y^{\prime}\right)\right\rangle d y^{\prime}, \\
& G(y)=\int_{\bar{y}}^{y} e^{\rho\left(y^{\prime}\right)} r\left(y^{\prime}\right)\left(2 r^{\prime}\left(y^{\prime}\right)-\rho^{\prime}\left(y^{\prime}\right) r\left(y^{\prime}\right)\right) \Theta\left(y^{\prime}\right) d y^{\prime} .
\end{aligned}
$$

The primes on a variable (e.g. $\rho^{\prime}$ and $r^{\prime}$ ) indicate the derivative with respect to the radial coordinate $y$. The constant $\bar{y}$ is the location of the horizon where $\rho \rightarrow-\infty$. The divergence of the energy-momentum tensor at the horizon is parametrized by the constant $q$ (with $H(y)+G(y)$ vanishing at $y=\bar{y})$, assuming that $\Theta(y)$ is finite there. ${ }^{1}$

For instance, in terms of $A(z), B(z)$ and the proper radial coordinate $z$ (see eq. (2.7)), $H$ and $G$ are

$$
\begin{aligned}
& H(z)=\frac{1}{4} \int_{\bar{z}}^{z} \frac{e^{A\left(z^{\prime}\right)}}{B\left(z^{\prime}\right)}\left(A^{\prime}\left(z^{\prime}\right)-\frac{B^{\prime}\left(z^{\prime}\right)}{B\left(z^{\prime}\right)}\right)\left\langle T_{\mu}^{\mu}\left(z^{\prime}\right)\right\rangle d z^{\prime} \\
& G(z)=-\int_{\bar{z}}^{z} \frac{e^{A\left(z^{\prime}\right)}}{B\left(z^{\prime}\right)}\left(A^{\prime}\left(z^{\prime}\right)-3 \frac{B^{\prime}\left(z^{\prime}\right)}{B\left(z^{\prime}\right)}\right) \Theta\left(z^{\prime}\right) d z^{\prime} .
\end{aligned}
$$

The metric for a static, spherically symmetric space-time can in principle be solved from the semi-classical Einstein equation (2.1) for an arbitrary assignment of $\left\langle T^{\theta}{ }_{\theta}\right\rangle$. In the following, we shall find the solution of the metric both perturbatively and nonperturbatively for arbitrary $\left\langle T^{\theta}{ }_{\theta}\right\rangle$. In the perturbative analysis, we find the first order

\footnotetext{
${ }^{1}$ In general, $r^{\prime}(y)$ and $\rho^{\prime}(y)$ could diverge as $y \rightarrow \bar{y}$ if $e^{\rho(y)} \rightarrow 0$ there. We assume that the integrands are still finite at $y=\bar{y}$, as in the case of the Schwarzschild metric.
} 
correction to the Schwarzschild metric. The non-perturbative analysis is carried out in a small neighborhood of an arbitrary point in space. Putting the perturbative and nonperturbative results together, we get a consistent picture of the black-hole geometry including the back reaction of the energy-momentum tensor of $4 \mathrm{D}$ conformal quantum fields.

\section{General perturbative solution in areal radius}

In the following, we will solve the first order perturbative correction to the Schwarzschild metric due to the energy-momentum tensor of $4 \mathrm{D}$ conformal quantum fields with full generality, and we will classify the solutions according to their near-horizon geometry. In this section, we shall use the areal radius $r$ as the coordinate parametrizing the radial direction. In the next two sections we will use other radial coordinates.

\subsection{Perturbative analysis}

Here we consider the ansatz (2.5) for the metric. The parametric functions $\rho(r)$ and $F(r)$ are expanded in powers of the Newton constant $\kappa$ as

$$
\begin{gathered}
\rho=\rho_{0}+\kappa \rho_{1}+\kappa^{2} \rho_{2}+\cdots, \\
F=F_{0}+\kappa F_{1}+\kappa^{2} F_{2}+\cdots,
\end{gathered}
$$

where $\rho_{n}$ and $F_{n}$ are of $\mathcal{O}\left(\kappa^{0}\right)$. Note that $[\kappa]=L^{2}$ and $\left[\rho_{1}\right]=\left[F_{1}\right]=L^{-2}$ in terms of the dimension of length $L$. The 0 -th order solution is given by the Schwarzschild metric:

$$
\begin{aligned}
& \rho_{0}=\log \left(1-\frac{a}{r}\right), \\
& F_{0}=\left(1-\frac{a}{r}\right)^{2},
\end{aligned}
$$

where $a$ is the Schwarzschild radius. We shall solve the semi-classical Einstein equation (2.1) perturbatively in the $\kappa$-expansion for the leading order perturbative correction $\rho_{1}, F_{1}$. We assume that the energy-momentum tensor comes from quantum effects and are of $\mathcal{O}\left(\kappa^{0}\right)$.

For any given radial pressure $\left\langle T^{r}{ }_{r}\right\rangle$, the metric correction $\rho_{1}$ and $F_{1}$ can then be directly solved from the semi-classical Einstein equations (2.12) and (2.11). Eq. (2.12) is

$$
\frac{F_{1}(r)}{(r-a)^{2}}+\frac{(r-a) \rho_{1}^{\prime}-\rho_{1}}{r^{2}}=\left\langle T_{r}^{r}\right\rangle .
$$

It allows one to express $F_{1}$ in terms of $\rho_{1}$ :

$$
F_{1}(r)=(r-a)^{2}\left[\left\langle T_{r}^{r}\right\rangle-\frac{(r-a) \rho_{1}^{\prime}-\rho_{1}}{r^{2}}\right] .
$$

Plugging this into the other equation (2.11), we turn it into a second order differential equation for $\rho_{1}$ only:

$$
(2 r-3 a)(r-a) \rho_{1}^{\prime \prime}+2(2 r-3 a) \rho_{1}^{\prime}=J(r)
$$

where

$$
J(r) \equiv r^{2}(4 r-3 a)\left\langle\left(T_{r}^{r}\right)^{\prime}\right\rangle+6 r(2 r-a)\left\langle T_{r}^{r}\right\rangle-2 r^{2}\left\langle T_{\mu}^{\mu}\right\rangle
$$


We can then solve $\rho_{1}$ from this second order differential equation as

$$
\rho_{1}(r)=C_{0}+\int_{r_{0}}^{r} d r^{\prime} \frac{1}{\left(r^{\prime}-a\right)^{2}}\left[C_{1}+\int_{r_{0}}^{r^{\prime}} d r^{\prime \prime} \frac{\left(r^{\prime \prime}-a\right)^{2} J\left(r^{\prime \prime}\right)}{\left(2 r^{\prime \prime}-3 a\right)\left(r^{\prime \prime}-a\right)}\right],
$$

with integration constants $C_{0}$ and $C_{1}$. After finding the solution of $\rho_{1}$, one can easily compute $F_{1}$ from eq. (3.6).

The first order corrections in the $\kappa$-expansion, $\rho_{1}(r)$ and $F_{1}(r)$, are now written in terms of $\left\langle T_{r}^{r}\right\rangle$ and $\left\langle T_{\mu}^{\mu}{ }_{\mu}\right.$. The trace of the energy-momentum tensor $\left\langle T^{\mu}{ }_{\mu}\right\rangle$ is given by (2.30) for the Schwarzschild background, while $\left\langle T^{r}{ }_{r}\right\rangle$ is expressed as (2.17) (with (2.31) and (2.32)) which diverges in general at $r=a$. Since this divergence is related to the coordinate singularity in the $(t, r)$-coordinates, we assume that $\left\langle T^{\theta}{ }_{\theta}\right\rangle$, which is invariant under the coordinate transformation in the $(t, r)$-directions, does not diverge at $r=a$. Under this assumption, it would be convenient to express the results in terms of $\left\langle T^{\theta}{ }_{\theta}\right\rangle$, or equivalently, of $\Theta$.

To calculate the energy-momentum tensor in the limit $r \rightarrow a$, we expand eqs. $(2.16),(2.17),(2.31)$ and $(2.32)$ in powers of $(r-a)$ and find

$$
\begin{aligned}
\left\langle T^{t}{ }_{t}(r \rightarrow a)\right\rangle & =-\frac{q}{a(r-a)}+\frac{q+6\left(c_{4}+a_{4}\right)}{a^{4}}-\left\langle T^{\theta}{ }_{\theta}(r \rightarrow a)\right\rangle, \\
\left\langle T^{r}{ }_{r}(r \rightarrow a)\right\rangle & =\frac{q}{a(r-a)}+\frac{-q+6\left(c_{4}+a_{4}\right)}{a^{4}}-\left\langle T^{\theta}{ }_{\theta}(r \rightarrow a)\right\rangle .
\end{aligned}
$$

We shall thus represent $\left\langle T_{r}^{r}\right\rangle$ (for all $r$, not only for $r$ close to $a$ ) as

$$
\left\langle T_{r}^{r}(r)\right\rangle=\frac{q}{a(r-a)}+f(r),
$$

where

$$
f(r) \equiv-\frac{q}{a r}+\frac{1}{r(r-a)}[H(r)+G(r)] .
$$

Here, $H(r)$ and $G(r)$ are given by (2.31) and (2.32), respectively. The function $f(r)$ is determined by $\left\langle T^{\theta} \theta\right\rangle$ and $q$, and it is regular at $r=a$ (assuming that $\left\langle T_{\theta \theta}(a)\right\rangle$ is finite). As the energy-momentum tensor is assumed to come from quantum effects, $q$ should be of $\mathcal{O}\left(\kappa^{0} a^{-2}\right)$.

The results (3.6) (3.9) are now expressed in terms of $f(r)$ as

$$
\begin{aligned}
\rho_{1}(r)= & C_{0}+\frac{C_{1}}{r-a}+W(r)-\frac{q}{4 a(r-a)}\left[2\left(7 a^{2}+8 a r-4 r^{2}\right)\right. \\
& \left.+a(3 a-2 r)\left(4 \log \left(\frac{r-a}{a}\right)+9 \log \left(\frac{3 a-2 r}{a}\right)\right)\right], \\
F_{1}(r)= & -(r-a)\left\{\frac{\left[-2 C_{0}(r-a)-4 C_{1}\right]}{2 r^{2}}\right. \\
& +\frac{q\left[7 a^{2}-2 r^{2}+13 a r+9 a(2 a-r) \log \left(\frac{2 r-3 a}{a}\right)+4 a(2 a-r) \log \left(\frac{r-a}{a}\right)\right]}{2 a r^{2}} \\
& \left.-(r-a) f(r)+\frac{1}{2 r^{2}}[V(r)-2(r-a) W(r)]\right\},
\end{aligned}
$$


where

$$
\begin{aligned}
W(r) & \equiv \int_{a}^{r} d r^{\prime \prime} \frac{1}{\left(r^{\prime \prime}-a\right)^{2}} \frac{V\left(r^{\prime \prime}\right)}{2}, \\
V(r) & \equiv-2 \int_{a}^{r} d r^{\prime} \frac{r^{\prime}\left(a-r^{\prime}\right)}{\left(3 a-2 r^{\prime}\right)}\left[6\left(a-2 r^{\prime}\right) f\left(r^{\prime}\right)+2 r^{\prime}\left\langle T_{\mu}^{\mu}\left(r^{\prime}\right)\right\rangle+r^{\prime}\left(3 a-4 r^{\prime}\right) f^{\prime}\left(r^{\prime}\right)\right] .
\end{aligned}
$$

There are two integration constants $C_{0}$ and $C_{1}$. The constant $C_{0}$ corresponds to a scaling of the time coordinate $t$; and $C_{1}$ to a shift of the Schwarzschild radius $a$. They are fixed by specifying the asymptotic conditions at $r \rightarrow \infty$ : the choice of $t$ in the asymptotically flat spacetime fixes $C_{0}$; and the asymptotic total energy (which depends on the choice of $f(r)$ ) determines $C_{1}$.

Eqs. (3.14) and (3.15) give the most general first order perturbation of the Schwarzschild metric for a 4D conformal matter field in any state according to the semiclassical Einstein equation (2.1). The energy-momentum tensor $\left\langle T_{\mu \nu}\right\rangle$ of any quantum state is specified by a function $f(r)$ and a constant $q$ through eq. (3.12), with the rest of the energy-momentum tensor determined through the Weyl anomaly and conservation law.

\subsubsection{Analysis in the limit $r \rightarrow \infty$}

Now we consider the solution (3.14)-(3.15) in the two limits $r \rightarrow \infty$ and $r \rightarrow a$. In the asymptotic region at large $r$, the asymptotic expressions of the metric is given by

$$
\begin{aligned}
& \rho_{1}(r) \simeq\langle\left.T^{\theta}{ }_{\theta}(\infty)\right\rangle r^{2}+\left(7 a\left\langle T^{\theta}{ }_{\theta}(\infty)\right\rangle+3 \gamma_{1}\right) r+S \log (r) \\
&+ {\left[C_{0}-2 q+\frac{9}{2} q \log (2)-\frac{13}{2} q \log (a)\right] } \\
&- {\left[\frac{6\left(c_{4}+a_{4}\right)}{5 a}+a q+\frac{3 a}{2}\left(9 a^{2}\left\langle T^{\theta}{ }_{\theta}(\infty)\right\rangle+4 \gamma_{0}+6 a \gamma_{1}\right)\right] \frac{\log (r)}{r} } \\
&+ \frac{1}{a}\left[C_{1}-\frac{24}{5 a}\left(c_{4}+a_{4}\right)-\frac{29}{4} a q-27 a^{3}\left\langle T^{\theta}{ }_{\theta}(\infty)\right\rangle-12 a \gamma_{0}-21 a^{2} \gamma_{1}\right. \\
&\left.-\frac{9}{4} a q \log (2)+\frac{13}{4} a q \log (a)\right] \frac{1}{r}+\mathcal{O}\left(\frac{1}{r^{2}}\right), \\
& F_{1}(r) \simeq\left(3 a\left\langle T^{\theta}{ }_{\theta}(\infty)\right\rangle+\gamma_{1}\right) r+S \log (r) \\
&+\left[C_{0}-\frac{6\left(c_{4}+a_{4}\right)}{5 a^{2}}-3 q+\frac{9}{2} q \log (2)-\frac{13}{2} q \log (a)-19 a^{4}\left\langle T^{\theta}{ }_{\theta}(\infty)\right\rangle-5 \gamma_{0}-10 a \gamma_{1}\right] \\
&-\left[\frac{36\left(c_{4}+a_{4}\right)}{5 a}+6 a q+69 a^{3}\left\langle T^{\theta}{ }_{\theta}(\infty)\right\rangle+24 a \gamma_{0}+42 a^{2} \gamma_{1}\right] \frac{\log (r)}{r} \\
&+\left[-2 a C_{0}+2 C_{1}-\frac{18\left(c_{4}+a_{4}\right)}{5 a}-\frac{9 a q}{4}+\kappa G_{1}\right. \\
&\left.-11 a^{3}\left\langle T^{\theta}{ }_{\theta}(\infty)\right\rangle-7 a \gamma_{0}-12 a^{2} \gamma_{1}-\frac{27 a q}{2} \log (2)+\frac{39 a q}{2} \log (a)\right] \frac{1}{r}+\mathcal{O}\left(\frac{1}{r^{2}}\right),
\end{aligned}
$$

where

$$
S \equiv \frac{12\left(c_{4}+a_{4}\right)}{5 a^{2}}+2 q+21 a^{2}\left\langle T_{\theta}^{\theta}(\infty)\right\rangle+6 \gamma_{0}+12 a \gamma_{1}
$$


The constant parameters $\gamma_{0}, \gamma_{1}$ and $G_{1}$ are defined by the large- $r$ expansion of $G(r)(2.32)$ as

$$
G(r) \simeq\left\langle T^{\theta}{ }_{\theta}(\infty)\right\rangle r^{2}+\gamma_{1} r+\frac{3\left(c_{4}+a_{4}\right)}{10 a^{2}}+\gamma_{0}+\frac{G_{1}}{r}+\mathcal{O}\left(1 / r^{2}\right)
$$

and $\left\langle T^{\theta}{ }_{\theta}(\infty)\right\rangle$ is the angular pressure in asymptotically flat region, $\left\langle T^{\theta}{ }_{\theta}(r \rightarrow \infty)\right\rangle$. As the anomaly (2.30) goes to 0 in the asymptotically flat region

$$
\left\langle T_{\mu}^{\mu}(r \rightarrow \infty)\right\rangle=0,
$$

the part independent of $r$ in $\left\langle T_{\mu \nu}(r \rightarrow \infty)\right\rangle$ represents a thermal equilibrium state at spatial infinity parametrized by $\left\langle T^{\theta}{ }_{\theta}(\infty)\right\rangle$.

\subsubsection{Analysis in the limit $r \rightarrow a$}

In the near-horizon limit $r \rightarrow a$, we parametrize $C_{0}$ and $C_{1}$ in terms of two parameters $\tilde{c}_{0}$ and $\tilde{c}_{1}$ defined by

$$
C_{0}=\tilde{c}_{0}-\frac{9}{2} q, \quad C_{1}=\frac{9}{2} a q+\tilde{c}_{1} .
$$

Here, $\left[C_{0}\right]=L^{-2}$ and $\left[C_{1}\right]=L^{-1}$ for the dimension of length $L$, and $\tilde{c}_{0} \sim \mathcal{O}\left(a^{-2}\right)$ and $\tilde{c}_{1} \sim \mathcal{O}\left(a^{-1}\right)$. Then the solution is approximated by

$$
\begin{aligned}
\rho_{1}(r)= & -\frac{q a}{(r-a)} \log \left(\frac{r-a}{a}\right)+\frac{\tilde{c}_{1}-q a}{(r-a)}+2 q \log \left(\frac{r-a}{a}\right)+\tilde{c}_{0} \\
& +\left[-\frac{5}{2} \frac{q}{a}-3 a f(a)+a\left\langle T_{\mu}^{\mu}(a)\right\rangle-\frac{1}{2} a^{2} f^{\prime}(a)\right](r-a)+\mathcal{O}\left((r-a)^{2}\right), \\
F_{1}(r)= & -\frac{2 q(r-a)}{a} \log \left(\frac{r-a}{a}\right)+\frac{2 \tilde{c}_{1}}{a^{2}}(r-a) \\
& +\left[\frac{6}{a^{2}} q \log \left(\frac{r-a}{a}\right)+\frac{\tilde{c}_{0}}{r^{2}}-\frac{4 \tilde{c}_{1}}{a^{3}}+f(a)\right](r-a)^{2}+\mathcal{O}\left((r-a)^{3}\right) .
\end{aligned}
$$

From these expressions we see that, in the near-horizon region, the perturbation at the leading order depends only on the Schwarzschild radius $a$ and the constant parameter $q$, but not on the conformal charges or the tangential pressure $\left\langle T_{\theta \theta}(a)\right\rangle$.

Clearly, the perturbation theory fails at $r=a$ where $\rho_{1}$ diverges, while it provides a good approximation at large $r$. To find out the range of $r$ where the perturbation theory works, we examine the order of magnitude of some geometric quantities at $r=a$ :

$$
\begin{aligned}
R & =-\kappa\left[\frac{5 q}{a^{2}} \log \left(\frac{r-a}{a}\right)+\mathcal{O}\left((r-a)^{0}\right)\right]+\mathcal{O}\left(\kappa^{2}\right), \\
R_{\mu \nu} R^{\mu \nu} & =\mathcal{O}\left(\kappa^{2}\right), \\
R_{\mu \nu \lambda \sigma} R^{\mu \nu \lambda \sigma} & =\frac{12 a^{2}}{r^{6}}+\kappa\left[\frac{2 q}{a^{4}} \log \left(\frac{r-a}{a}\right)+\mathcal{O}\left((r-a)^{0}\right)\right]+\mathcal{O}\left(\kappa^{2}\right) .
\end{aligned}
$$

Noting $q=\mathcal{O}\left(a^{-2}\right)$, the quantum correction of $R_{\mu \nu \lambda \sigma} R^{\mu \nu \lambda \sigma}$ is sufficiently small as long as

$$
r-a \gg a e^{-a^{2} / \kappa} \text {. }
$$


Therefore, the perturbative expansion for the geometry is expected to be valid in this region. On the other hand, the perturbative corrections for $\rho$ and $F$, as is shown in (3.24) and (3.25), become comparable to the leading order terms around the region

$$
r-a \sim \mathcal{O}\left(\frac{\kappa}{a}\right) .
$$

This implies that the expressions (3.24) and (3.25) are valid only for

$$
r-a \gtrsim \mathcal{O}\left(\frac{\kappa}{a}\right),
$$

since they are calculated by assuming that $r-a \sim \mathcal{O}\left(\kappa^{0} a\right)$. The quantum corrections for geometric quantities above are all of $\mathcal{O}(\kappa \log \kappa)$ or smaller in (3.31) so we expect that the perturbation theory is valid in the range defined by eq. (3.31).

Within the range of validity of the perturbation theory, the quantum correction is most significant around the region (3.30) although it can in principle be even larger in the non-perturbative domain. The energy-momentum tensor (3.10) and (3.11) around the region (3.30) is estimated to be

$$
\left|\left\langle T^{t}{ }_{t}\right\rangle\right| \sim\left|\left\langle T_{r}^{r}\right\rangle\right| \sim \mathcal{O}\left(\frac{1}{\kappa a^{2}}\right) .
$$

This is what one would expect, according to the Einstein equation, of the energy-momentum tensor for a spacetime region in which the curvature is of $\mathcal{O}\left(1 / a^{2}\right){ }^{2}$ On the other hand, in comparison with the classical vacuum energy (which is exactly zero), the quantum vacuum energy is relatively high. For a black hole of a few solar mass, the energy density can be as high as $\mathcal{O}\left(\mathrm{MeV}^{4}\right)$.

\subsection{Classification of solutions}

To understand the quantum-corrected near-horizon geometry in more details, we examine the perturbative solution in the neighborhood (3.31). According to (3.3) and (3.24), we find

$$
\rho=\rho_{0}+\kappa \rho_{1}+\cdots=\left[1-\frac{\kappa a q}{(r-a)}\right] \log \left(1-\frac{a}{r}\right)+\cdots,
$$

and using (3.4) and (3.25), we find

$$
F=F_{0}+\kappa F_{1}+\cdots=\frac{(r-a)^{2}}{a^{2}}\left[1-\frac{2 \kappa a q}{(r-a)} \log \left(\frac{r-a}{a}\right)\right]+\cdots .
$$

These expressions determine the near-horizon geometry to the first order in the perturbation theory. The nature of the near-horizon geometry depends on the sign of the parameter $q$. The Hartle-Hawking vacuum corresponds to the condition $q=0$, while $q$ would be negative in the Boulware vacuum, as we have seen in an example in section 2.2. We consider the three possibilities: $q<0, q=0$ and $q>0$, separately in the following.

\footnotetext{
${ }^{2}$ In a self-consistetn model [26-32], (3.32) is obtained.
} 


\subsubsection{Wormhole-like throat $(q<0)$}

If $q<0$, in the limit $r \rightarrow a$ (moving towards $r=a$ from distance), we have

$$
\begin{aligned}
& \rho \simeq \rho_{0}+\kappa \rho_{1} \simeq\left[1+\frac{\kappa|q| a}{r-a}\right] \log \left(\frac{r-a}{a}\right)+\cdots, \\
& F \simeq F_{0}+\kappa F_{1} \simeq \frac{1}{a^{2}}(r-a)^{2}\left[1+\frac{2 \kappa|q| a}{r-a} \log \left(\frac{r-a}{a}\right)\right]+\cdots .
\end{aligned}
$$

The perturbation theory breaks down when $r$ is too close to the Schwarzschild radius:

$$
r-a \ll \kappa|q| a \text {. }
$$

Hence the existence of the horizon at $r=a$ is not guaranteed.

According to this approximation, $F$ has a zero at $r>a$, implying that there is a local minimum in $r$ outside the horizon. ${ }^{3}$ We refer to this local minimum in $r$ as the "neck" or "throat" of a "wormhole-like structure", as it resembles the geometry of a traversable wormhole, although it does not lead to another open spacetime. Noting eq. (2.6), the location of the wormhole neck is where $d r / d z=0$, i.e. where

$$
F(r)=0
$$

With $F(r)$ approximated by $F_{0}+\kappa F_{1}, r-a$ is of $\mathcal{O}(\kappa / a)$ at the neck. But this means that the 0 -th order, 1 st order and 2 nd order terms in $F$ are all of $\mathcal{O}\left(\kappa^{2} / a^{4}\right)$. Hence it is not reliable to determine the location of the neck based the first order perturbation in the $r$-coordinate.

In section 4, we will see that the perturbation theory in another coordinate system (using the proper radial coordinate $z$ ) allows us to locate the neck with better accuracy. In section 6.2 , we will further confirm this perturbative result by non-perturbative analysis.

\subsubsection{Event horizon $(q=0)$}

When $q=0$, we have from eqs. (3.14) and (3.15)

$$
\begin{aligned}
& \rho_{1} \simeq-\frac{1}{(r-a)} \Delta a+\cdots, \\
& F_{1} \simeq-\frac{2}{a^{2}}(r-a) \Delta a+\cdots,
\end{aligned}
$$

where

$$
\Delta a \equiv C_{1}+3\left(c_{4}+a_{4}\right) \frac{(5+2 \log (a))}{5 a} .
$$

These expressions coincide with the perturbation of the Schwarzschild metric for a shift of the Schwarzschild radius by

$$
a \rightarrow a+\Delta a
$$

This means that the black-hole mass receives a quantum correction $\Delta a / 2$. We can redefine the Schwarzschild radius $a$ (tune $C_{1}$ such that $\Delta a=0$ ) to absorb this correction. As a

\footnotetext{
${ }^{3}$ To claim that the neck is a local minimum of $r$, we have checked not only that $F=0$ at the neck, but also that $\left.\frac{d F}{d r}\right|_{\text {neck }}>0$.
} 
result, $\rho_{1}$ and $F_{1}$ are only modified at higher orders of the $(r-a)$-expansion in the nearhorizon region, and the geometry of the Schwarzschild horizon is not significantly modified by the quantum effect. Only in this case, the perturbation theory is valid down to the horizon at $r=a$.

\subsubsection{No neck \& no horizon $(q>0)$}

If $q>0$, in the limit $r \rightarrow a$ (moving towards $r=a$ from distance), we have

$$
\begin{aligned}
& \rho \simeq \rho_{0}+\kappa \rho_{1} \simeq\left[1-\frac{\kappa|q| a}{r-a}\right] \log (r-a)+\cdots, \\
& F \simeq F_{0}+\kappa F_{1} \simeq \frac{1}{a^{2}}(r-a)^{2}\left[1-\frac{2 \kappa|q| a}{r-a} \log (r-a)\right]+\cdots,
\end{aligned}
$$

from eqs. (3.33) and (3.34). In contrast with the case $q<0$, there is no local minimum of $r$ outside the Schwarzschild radius, because $F$ has no zero for $r>a$, while the perturbation theory breaks down in the region

$$
r-a \ll \kappa|q| a .
$$

Furthermore, since $\rho_{1} \rightarrow+\infty$ in the limit $r \rightarrow a$, the event horizon is removed as $\rho$ no longer approaches to $-\infty$, On the other hand, since the perturbation theory does not apply to the immediate neighborhood of the horizon at $r=a$, we cannot rule out the existence of a horizon beyond the range of validity of the perturbation theory.

\subsection{Higher order corrections}

It is straightforward to calculate the second order corrections to $\rho$ and $F$. The second order solutions $\rho_{2}$ and $F_{2}$ are calculated by solving the second order semi-classical Einstein equation and expanded around $r=a$ as

$$
\begin{aligned}
\rho_{2}(r)= & \frac{1}{2(r-a)^{2}}\left\{a^{2} q^{2}-\tilde{c}_{1}^{2}+2 a \tilde{c}_{1} q \log \left(\frac{r-a}{a}\right)-a^{2} q^{2}\left[\log \left(\frac{r-a}{a}\right)\right]^{2}\right\} \\
& +\mathcal{O}\left((r-a)^{-1}\right), \\
F_{2}(r)= & \frac{\tilde{c}_{1}^{2}}{a^{2}}-\frac{2 \tilde{c}_{1} q}{a}+2 q^{2}-\frac{2 q}{a}\left(\tilde{c}_{1}-a q\right) \log \left(\frac{r-a}{a}\right)+q^{2}\left[\log \left(\frac{r-a}{a}\right)\right]^{2} \\
& +\mathcal{O}((r-a)) .
\end{aligned}
$$

For $r \sim \mathcal{O}(\kappa / a)$, they are of $\mathcal{O}\left(\kappa^{-2}\right)$ and $\mathcal{O}\left(\kappa^{0} a^{-4}\right)$ respectively. Then, the leading, first, and second order terms of the perturbative expansion, (3.1) and (3.2), have the same order of magnitude, $\mathcal{O}\left(\kappa^{0} a^{0}\right)$ for $\rho$ and $\mathcal{O}\left(\kappa^{2} a^{-4}\right)$ for $F$, respectively. This is because the expressions (3.24), (3.25), (3.46) and (3.47) are calculated under the assumption that $r-a \sim \mathcal{O}\left(\kappa^{0} a\right)$, and hence, the higher order corrections become comparable to the lower order terms for $r-a \sim \mathcal{O}\left(\kappa / a^{2}\right)$. This does not imply the breakdown of the perturbative expansion and the expansion would be valid if it is calculated by using the appropriate assumption, $r-a \sim \mathcal{O}\left(\kappa / a^{2}\right)$. We will discuss this issue again in the subsequent sections. 


\subsection{Time-dependent perturbations}

In the above, we have focused on static configurations so that the off-diagonal terms $\left\langle T^{t}{ }_{r}\right\rangle$ (2.18) and $\left\langle T^{r}{ }_{t}\right\rangle$ (2.20) can be set to zeros by setting $k=0$. When $k \neq 0$ in eqs. (2.18) and (2.20), the energy-momentum tensor is still time-independent but we need to consider a time-dependent perturbation of the metric. We can still use eq. (2.5) as the ansatz for the metric, but $\rho_{1}$ and $F_{1}$ are now time-dependent.

It is straightforward to solve the perturbation of the metric when $k$ is turned on. The $k$-dependent terms of the energy-momentum tensor are

$$
\begin{aligned}
\left\langle T^{t}{ }_{t}\right\rangle & =\left\langle T_{r}^{r}\right\rangle=\left\langle T_{\theta}^{\theta}\right\rangle=\left\langle T_{\phi}^{\phi}\right\rangle=0, \\
\left\langle T^{t}{ }_{r}\right\rangle & =\frac{k}{(r-a)^{2}} .
\end{aligned}
$$

The corresponding solution is

$$
\begin{aligned}
\rho_{1} & =\frac{k t}{r-a}, \\
F_{1} & =\frac{2 k(r-a) t}{r^{2}} .
\end{aligned}
$$

For the most general time-independent conserved tensor (2.16)-(2.21) in the Schwarzschild background, the solution of the metric perturbation is thus a superposition of the previous result (3.14)-(3.15) for time-independent metric and the time-dependent part (3.50)-(3.51).

\section{General perturbative solution in proper radial coordinate}

In this section, we repeat the derivation of the metric perturbation due to a generic energymomentum tensor of $4 \mathrm{D}$ conformal quantum fields, but in terms of the proper radial coordinate $z$, which measures the proper distance along the radial direction. We will see that certain features of the near-horizon geometry which is obscured in the $r$-coordinate is more manifest in the $z$-coordinate.

The ansatz of the metric is eq. (2.7). The 0 -th order terms are given by the Schwarzschild solution:

$$
\begin{aligned}
& B_{0}=r_{0}^{2}(z), \\
& A_{0}=\log \left[\left(1-\frac{a}{r_{0}(z)}\right) r_{0}^{4}(z)\right],
\end{aligned}
$$

where $r_{0}(z)$ is defined through eq. (2.6) by

$$
\frac{d r_{0}(z)}{d z}=\sqrt{1-\frac{a}{r_{0}(z)}} .
$$

This equation is invariant under a shift of $z$, so we can simply choose $z$ such that $z=0$ at the horizon, i.e. $r_{0}(z=0)=a$. The function $r_{0}(z)$ can then be expanded around $z=0$ as

$$
r_{0}(z)=a+\frac{z^{2}}{4 a}-\frac{z^{4}}{48 a^{3}}+\frac{11 z^{6}}{2880 a^{5}}+\cdots .
$$


The 0 -th order solutions to $B(z)(4.1)$ and $A(z)$ (4.2) can also be expanded around $z=0$ as

$$
\begin{aligned}
& B_{0}(z)=a^{2}+\frac{z^{2}}{2}+\frac{z^{4}}{48 a^{2}}-\frac{z^{6}}{360 a^{4}}+\cdots \\
& A_{0}(z)=\log \left(\frac{a^{2} z^{2}}{4}\right)+\frac{2 z^{2}}{3 a^{2}}-\frac{13 z^{4}}{90 a^{4}}+\cdots
\end{aligned}
$$

To solve the semi-classical Einstein equation (2.1) for the perturbative expansion

$$
\begin{aligned}
& B=B_{0}+\kappa B_{1}+\cdots, \\
& A=A_{0}+\kappa A_{1}+\cdots,
\end{aligned}
$$

we first solve for $A_{1}^{\prime}(z)$ algebraically in terms of $B_{1}(z), q$ and $f(z)$ from $G^{z}{ }_{z}=\kappa\left\langle T^{z}{ }_{z}\right\rangle{ }^{4}$ One can then use the expression of $A_{1}^{\prime}(z)$ to turn $G^{\mu}{ }_{\mu}=\kappa\left\langle T^{\mu}{ }_{\mu}\right\rangle$ into an equation for $B_{1}(z)$ only, and $B_{1}(z)$ can be solved, at least as an expansion of $z$. In fact, it would be difficult to solve the first order equations exactly, and hence, we will focus on the behavior around $z=0$ and use the expansions (4.5) and (4.6).

The general solutions to the first order semi-classical Einstein equations are

$$
\begin{aligned}
B_{1}= & 4 a^{2} q \log \left(\frac{z}{a}\right)+B_{10}+B_{11}\left(\frac{z}{a}+\frac{z^{3}}{12 a^{3}}+\cdots\right) \\
& +\frac{a^{2}}{6}\left[5 q+3 a^{2} f(0)\right]\left(\frac{z}{a}\right)^{2}+\cdots \\
A_{1}= & \frac{B_{11}}{a^{2}}\left(\frac{2 a}{z}+\frac{4 z}{3 a}+\cdots\right)+8 q\left[1-\frac{1}{3}\left(\frac{z}{a}\right)^{2}\right] \log \left(\frac{z}{a}\right)+A_{10} \\
& +\frac{1}{9 a^{2}}\left[-6 B_{10}+4 a^{2} q+18\left(c_{4}+a_{4}\right)+3 a^{4} f(0)\right]\left(\frac{z}{a}\right)^{2}+\cdots
\end{aligned}
$$

There are three integration constants $A_{10}, B_{10}$ and $B_{11}$ in the general solution for the first order perturbation. $A_{10}$ can always be set to 0 by rescaling the time coordinate $t$. $B_{10}$ corresponds to a shift of the Schwarzschild radius $a$, and thus can be absorbed by a redefinition of $a$. $B_{11}$ corresponds to a shift of the $z$-coordinate. In the following, we put $B_{10}=B_{11}=A_{10}=0$.

Unless $q=0$, we have $B_{1}(z) \rightarrow \pm \infty$ as $z \rightarrow 0$, and the perturbation theory is valid only in the region sufficiently far away from the point $z=0$. The near-horizon geometry can be classified into three categories depending on whether $q<0, q=0$ or $q>0$, as we have seen in the previous section. We consider each case separately in the following.

\subsection{Wormhole-like throat $(q<0)$}

Let us now focus on the case $q<0$. We have seen in section 3.2.1 that there is a wormholelike throat in this case, i.e. a local minimum of the areal radius $r$, or equivalently, a local

\footnotetext{
${ }^{4}$ Note that $\left\langle T_{z}^{z}\right\rangle=\left\langle T_{r}^{r}\right\rangle$, so we can use eq. (3.12) to set

$$
\left\langle T_{z}^{z}(z)\right\rangle=\frac{q}{a\left(r_{0}(z)-a\right)}+f(z)
$$
}

for an arbitrary smooth function $f(z)$. 
minimum of $B(z)$. We will see that the perturbative analysis in the $z$-coordinate for this case better displays details about the near-horizon geometry. On the other hand, the perturbative analysis in the $r$-coordinate in section 3.2.1 has the advantage that the first order solutions can be obtained without using the expansion in the radial coordinate, and hence, the integration constants around $r=a$ can be related to those in the asymptotically flat region.

The throat is located where $B \simeq B_{0}+\kappa B_{1}$ has a vanishing first derivative. The condition $B^{\prime}\left(z_{0}\right)=0$ implies that

$$
z \equiv z_{0} \simeq \sqrt{-4 \kappa a^{2} q}+\cdots
$$

Clearly, the wormhole throat exists only if $q<0$.

Assuming that $q<0$, we expand $B$ and $A$ around the neck at $z=z_{0}$ and find

$$
\begin{aligned}
& B(z) \simeq a^{2}+\kappa\left[B_{10}-2 a^{2} q+4 a^{2} q \log \left(\frac{z_{0}}{a}\right)\right]+\left(z-z_{0}\right)^{2}+\mathcal{O}\left(\kappa^{3 / 2}\right) \\
& A(z) \simeq \log (\kappa q)+\kappa\left[-\frac{8 q}{3}+8 q \log \left(\frac{z_{0}}{a}\right)\right]+\left[\frac{1}{a \sqrt{-\kappa q}}-\frac{4 \sqrt{-\kappa q}}{3 a}\right]\left(z-z_{0}\right)+\mathcal{O}\left(\kappa^{3 / 2}\right),
\end{aligned}
$$

for the neighborhood of $z=z_{0}$ in which

$$
\left|z-z_{0}\right| \lesssim \mathcal{O}\left(\kappa^{1 / 2}\right)
$$

The expansion of $A(z)$ is singular in the limit $\kappa a^{2} q \rightarrow 0$ unless the constraint (4.15) is imposed.

The second term of $B(z)$ (4.13) can always be absorbed in the first term $a^{2}$ by a redefinition of $a$. The expansion of $B(z)$ in powers of $\left(z-z_{0}\right)$ is free of the linear term, as $z_{0}$ is chosen to be the point of local extremum. Note that the coefficient of the quadratic term $\left(z-z_{0}\right)^{2}$ is always 1 (up to $\mathcal{O}(\kappa)$ correction) independent of all parameters. We will see below that this feature persists in the non-perturbative analysis.

The first three terms (the constant part) of $A(z)$ can always be set to 0 by a scaling of the time-coordinate $t$. Note that the conformal charges $c_{4}, a_{4}$ do not appear in eq. (4.13) nor (4.14). In terms of the radial distance coordiante $z$, the conformal anomaly has little effect on the near-horizon geometry when $q<0$.

\subsection{Event horizon $(q=0)$}

For the case $q=0$, the solution is approximately

$$
\begin{aligned}
& B=a^{2}+\frac{z^{2}}{2}+\kappa \frac{a^{2}}{2} f(0) z^{2}+\cdots, \\
& A=2 \log \left(\frac{a z}{2}\right)+\frac{2 z^{2}}{3 a^{2}}+\kappa\left[\frac{2\left(c_{4}+a_{4}\right)}{a^{4}}+\frac{f(0)}{3}\right] z^{2}+\cdots
\end{aligned}
$$

according to eqs. (4.5), (4.6), (4.10) and (4.11). Since the first order correction of $\mathcal{O}(\kappa)$ starts only at $\mathcal{O}\left(z^{2}\right)$, so the near-horizon geometry is only slightly modified in a small neighborhood of $z=0$. It is essentially the same as the Schwarzschild spacetime. This is in agreement with the result in section 3.2.2 which was obtained in terms of the $r$-coordinate. 


\subsection{No neck \& no horizon $(q>0)$}

For $q>0$, we derive from eqs. (4.5), (4.6), (4.10) and (4.11) the first order perturbation near the horizon:

$$
\begin{aligned}
& B(z) \simeq a^{2}+\frac{z^{2}}{2}+4 \kappa a^{2} q \log \left(\frac{z}{a}\right)+\cdots, \\
& A(z) \simeq 2 \log \left(\frac{a z}{2}\right)+\frac{2 z^{2}}{3 a^{2}}+8 \kappa q \log \left(\frac{z}{a}\right)+\cdots .
\end{aligned}
$$

According to these expressions, the areal radius has no local minimum and it vanishes somewhere around $z \sim a e^{-1 /(4 \kappa q)}$, where $A(z) \sim \mathcal{O}(1 /(\kappa q))$ and $B(z) \sim 0$ for $a^{2} \gg \kappa$. Strictly speaking, the perturbation theory is valid only for

$$
z \gg a e^{-1 /(4 \kappa q)},
$$

but it shows that the horizon can only exist, if it does exist, in the microscopic range of the areal radius.

What we have seen in this section is that, in the large-scale geometry, there is no horizon for both $q<0$ and $q>0$. The horizon exists only when $q$ is fine-tuned to exactly 0 . This result is also compatible with the calculation in the $r$-coordinate in section 3.2.3.

\subsection{Higher order corrections}

We have seen that the geometry near the horizon is modified by perturbative corrections. This happens because the perturbative expansion around the Schwarzschild solution is calculated by assuming $r-a \sim \mathcal{O}\left(\kappa^{0}\right)$, or equivalently, $z \sim \mathcal{O}\left(\kappa^{0}\right)$, and hence, higher order terms can be comparable to lower order terms if $z$ is sufficiently small as $z^{2} \sim \mathcal{O}(\kappa)$. More explicitly, perturbative expansion of $A(z)$ and $B(z)$ are expressed as

$$
\begin{aligned}
& A(z)=\log \left(\frac{z^{2}}{4 a^{2}}\right)+\frac{2 z^{2}}{3 a^{2}}+8 \kappa q \log \left(\frac{z}{a}\right)+\cdots, \\
& B(z)=a^{2}+\frac{z^{2}}{2}+4 a^{2} \kappa q \log \left(\frac{z}{a}\right)+\cdots .
\end{aligned}
$$

The first and second terms of the expressions above come from the 0 -th order terms of the perturbative expansion and the third terms are first-order corrections. If $z$ is small as $z^{2} \sim \mathcal{O}(\kappa)$, only the first terms give $\mathcal{O}\left(\kappa^{0}\right)$ contributions but both the second and third terms become of $\mathcal{O}(\kappa)$. For $q<0$, the local minimum of $B(z)$ moves to the outside of the horizon because the second term and third term in eq. (4.22) are comparable in this region. The structure around the local minimum might be modified if the higher order terms in eq. (4.22) become comparable to these terms when $z^{2} \sim \mathcal{O}(\kappa)$. In the following, we will calculate the second order corrections and show that they do not modify the structure of the neck at leading order.

We consider the perturbative expansion to the second order

$$
\begin{aligned}
& B=B_{0}+\kappa B_{1}+\kappa^{2} B_{2}+\cdots, \\
& A=A_{0}+\kappa A_{1}+\kappa^{2} A_{2}+\cdots .
\end{aligned}
$$


The general solutions for $B_{2}$ and $A_{2}$ are calculated from the second order terms of the semi-classical Einstein equation as

$$
\begin{aligned}
& B_{2}=B_{20}-4 a^{2} q^{2} \log z+B_{21} \frac{z}{a}+\mathcal{O}\left(z^{2}\right), \\
& A_{2}=\frac{B_{21}}{a^{2}}\left(\frac{2}{z}+\frac{4 z}{3 a}\right)-16 q^{2}(\log z)^{2}+A_{20}+\mathcal{O}\left(z^{2}\right) .
\end{aligned}
$$

The integration constants $A_{20}, B_{20}$ and $B_{21}$ can be set to zero by rescaling the time coordinate $t$, shifting the Schwarzschild radius $a$ and shifting the $z$-coordinates, respectively, as for those at the first order. Then, for $z^{2} \sim \mathcal{O}(\kappa)$, the above expressions have only $\mathcal{O}\left(\kappa^{0}\right)$ terms, which give contributions of $\mathcal{O}\left(\kappa^{2}\right)$ to $B$ and $A$. Therefore, the expressions (4.21) and (4.22) are sufficient to determine the behavior of $A$ and $B$ up to $\mathcal{O}\left(\kappa^{2}\right)$.

In order to see why the perturbative expansion for $\rho$ and $F$ in terms of $r$-coordinate is not good for $r-a \sim \mathcal{O}(\kappa)$, we write the proper radial coordinate $z$ as a function of $r$. From eq. (4.22), $z^{2}$ is expressed as

$$
z^{2}=2\left(r^{2}-a^{2}\right)-4 a^{2} \kappa q \log \left(\frac{r^{2}-a^{2}}{a^{2}}\right)-\frac{16 a^{4} \kappa^{2} q^{2}}{r^{2}-a^{2}} \log \left(\frac{r^{2}-a^{2}}{a^{2}}\right)+\mathcal{O}\left(\kappa^{3}\right),
$$

assuming that $r-a \sim \mathcal{O}\left(\kappa^{0}\right)$. As the expression above is calculated from the first 3 terms in eq. (4.22), which give $\mathcal{O}(\kappa)$ contributions for $z^{2} \sim \mathcal{O}(\kappa)$, all terms in eq. (4.27), including higher order terms, become $\mathcal{O}(\kappa)$ and comparable. This implies that the perturbative expansion in terms of $r$ (which is calculated assuming $r-a \sim \mathcal{O}\left(\kappa^{0}\right)$ ) does not give a good expansion for $r-a \sim \mathcal{O}(\kappa)$, and higher order terms give contributions of the same order.

\section{Perturbative solution near Schwarzschild radius}

In the previous sections, we have studied perturbations around the Schwarzschild solution. In general, the first-order correction becomes very large near the Schwarzschild radius $r=a$, implying that the perturbative expansion around the Schwarzschild solution is not good near the Schwarzschild radius. However, the perturbative expansion there is simply the small- $\kappa$ expansion for the semi-classical Einstein equation. Assuming that the semiclassical Einstein equation has a solution for arbitrary $\kappa$, the perturbative expansion should be possible at arbitrary $r$. This implies that the zero-th order solution in the perturbative expansion is not given by the Schwarzschild solution near the Schwarzschild radius. In this section, we focus on the solution near the Schwarzschild radius and study the perturbative expansion there.

The perturbative expansion around the Schwarzschild solution is valid for

$$
r-a \gg \frac{\kappa}{a}
$$

but it is not good if $r$ is very close to the Schwarzschild radius as

$$
r \sim a+\frac{\kappa}{a}
$$


and hence we consider the perturbative expansion in this region. In order to focus on this region, we introduce the tortoise coordinate $x$ as

$$
d x^{2}=\frac{d r^{2}}{F(r)}=e^{-\rho(x)} d z^{2},
$$

and then, the metric is expressed as

$$
d s^{2}=-e^{\rho(x)}\left[d t^{2}-d x^{2}\right]+r^{2}(x) d \Omega^{2} .
$$

We focus on the geometry near $r=a$. In eq. (5.1), where the expansion around the Schwarzschild solution is good, $e^{\rho(x)}$ is given by

$$
e^{\rho(x)}=1-\frac{a}{r(x)},
$$

and approaches to $\mathcal{O}(\kappa)$ as $r$ goes to $r=a+\mathcal{O}(\kappa)$. For the junction condition for the expansion in (5.1) and that in (5.2), $e^{\rho(x)}$ must behave as

$$
e^{\rho(x)}=\mathcal{O}(\kappa) .
$$

Therefore, in (5.2), $\rho(x)$ and $r(x)$ should be expanded as

$$
\begin{aligned}
e^{\rho(x)} & =\kappa e^{\tilde{\rho}_{0}(x)+\kappa \rho_{1}(x)}+\cdots, \\
r(x) & =a+\kappa r_{1}(x)+\cdots .
\end{aligned}
$$

We solve the semi-classical Einstein equation, (2.11) and (2.12), in the expansion above to first order, $\rho_{1}(x)$ and $r_{1}(x)$. The trace part of the energy-momentum tensor is given by the trace anomaly (2.2), while $\left\langle T^{x}{ }_{x}\right\rangle$ is given by (2.36). Near the Schwarzschild radius, they are expressed in terms of the expansion above as

$$
\begin{aligned}
\left\langle T^{\mu}{ }_{\mu}\right\rangle & =\frac{c_{4}}{3 \kappa^{2}} e^{-2 \tilde{\rho}_{0}(x)} \tilde{\rho}_{0}^{\prime \prime 2}(x)+\mathcal{O}\left(\kappa^{-1}\right), \\
\left\langle T^{x}{ }_{x}\right\rangle & =\frac{q e^{-\tilde{\rho}_{0}(x)}}{\kappa a^{2}}+\mathcal{O}\left(\kappa^{0}\right) .
\end{aligned}
$$

Here, we assumed that $\left\langle T^{\theta}{ }_{\theta}\right\rangle$ does not diverge as $e^{\rho(x)} \rightarrow 0$, and hence, $H(x)$ and $G(x)$ are of $\mathcal{O}(\kappa)$.

The leading order term of (2.11), which is of $\mathcal{O}\left(\kappa^{-1}\right)$, is calculated as

$$
e^{-\tilde{\rho}_{0}(x)} \tilde{\rho}_{0}^{\prime \prime}(x)=\frac{c_{4}}{3} e^{-2 \tilde{\rho}_{0}(x)} \tilde{\rho}_{0}^{\prime \prime 2}(x),
$$

and a solution is given by

$$
e^{\tilde{\rho}_{0}(x)}=\tilde{c}_{0} e^{\lambda_{0} x}
$$

where $c_{0}$ and $\lambda_{0}$ are integration constants. There is another solution which is the solution of

$$
\tilde{\rho}_{0}^{\prime \prime}(x)-c_{4} e^{\tilde{\rho}_{0}(x)}=0,
$$

which gives large curvature of $\mathcal{O}\left(\kappa^{-1}\right)$, and should be excluded as an unphysical solution. 
By substituting the first order solution (5.12), the next-to-leading order terms of the semi-classical Einstein equation (2.11) and (2.12) give the following differential equations for $\rho_{1}(x)$ and $r_{1}(x)$ :

$$
\begin{aligned}
& 0=\tilde{c}_{0} e^{\lambda_{0} x}+q-a \lambda_{0} r_{1}^{\prime}(x), \\
& 0=-2 \tilde{c}_{0} e^{\lambda_{0} x}+a^{2} \rho_{1}^{\prime \prime}(x)+4 a r_{1}^{\prime \prime}(x),
\end{aligned}
$$

and they are solved as

$$
\begin{aligned}
& \rho_{1}(x)=\bar{\rho}_{1}+\lambda_{1} x-\frac{2 \tilde{c}_{0} e^{\lambda_{0} x}}{a^{2} \lambda_{0}^{2}}, \\
& r_{1}(x)=a_{1}+\frac{\tilde{c}_{0} e^{\lambda_{0} x}+\lambda_{0} q x}{a \lambda_{0}^{2}},
\end{aligned}
$$

where $a_{1}, \bar{\rho}_{1}$ and $\lambda_{1}$ are integration constants, which can be absorbed by redefinitions of $a, \tilde{c}_{0}$ and $\lambda_{0}$, respectively. The solution to the first order perturbation is obtained as

$$
\begin{aligned}
& e^{\rho(x)}=\kappa \tilde{c}_{0} e^{\lambda_{0} x}\left(1-\frac{2 \kappa \tilde{c}_{0} e^{\lambda_{0} x}}{a^{2} \lambda_{0}^{2}}\right)+\mathcal{O}\left(\kappa^{3}\right), \\
& r(x)=a+\kappa \frac{\tilde{c}_{0} e^{\lambda_{0} x}+\lambda_{0} q x}{a \lambda_{0}^{2}}+\mathcal{O}\left(\kappa^{2}\right) .
\end{aligned}
$$

\subsection{Wormhole-like throat $(q<0)$}

If $q<0, r$ has a local minimum as we have seen in the previous sections. From eq. (5.19), the local minimum is located at

$$
x=x_{0} \equiv \frac{1}{\lambda_{0}} \log \frac{|q|}{\tilde{c}_{0}}
$$

and $r$ is expanded as

$$
r(x) \simeq a+\frac{\kappa q}{a \lambda_{0}^{2}}\left(-1+\log \frac{|q|}{\tilde{c}_{0}}\right)+\frac{\kappa|q|}{2 a}\left(x-x_{0}\right)^{2}+\cdots .
$$

In terms of the proper radial coordinate, $B(z)=r^{2}(z)$ is expanded as

$$
B(z) \simeq a^{2}+\left(z-z_{0}\right)^{2}+\cdots,
$$

where we redefined $a$ again to absorb the $\mathcal{O}(\kappa)$ constant term above. This is consistent with the result (4.13) in the previous section.

\subsection{Event horizon $(q=0)$}

For $q=0, r$ is given by

$$
r(x)=a+\frac{\kappa \tilde{c}_{0} e^{\lambda_{0} x}}{a \lambda_{0}^{2}}+\mathcal{O}\left(\kappa^{2}\right) .
$$

In this case, $r$ does not have local minimum but approaches to $r=a$ as $x \rightarrow-\infty$. The event horizon is located in the limit $x \rightarrow-\infty$ in the tortoise coordinate for classical solution and hence this behavior is consistent with the event horizon. In the proper radial coordinate, $B(z)=r^{2}(z)$ is expanded as

$$
B(z)=a^{2}+\frac{1}{2} z^{2}+\cdots,
$$

which is also consistent with the result in the previous section, (4.16). 


\subsection{No neck \& no horizon $(q>0)$}

For $q>0,(5.19)$ is a monotonic increasing function and has no local minimum. The areal radius $r$ can be smaller than $a$ but $e^{\rho(x)}$, whose leading order term is given by (5.12), approaches to zero but never goes to zero, at least in this near-Schwarzschild-radius region. Therefore, there are no neck or event horizon. In terms of the proper radial coordinate $z$, $B(z)$ is expressed as

$$
B(x)=a^{2}+\frac{1}{2} z^{2}+\frac{4 \kappa q}{\lambda_{0}^{2}} \log \frac{\lambda_{0} z}{2}+\mathcal{O}\left(\kappa^{2}\right) .
$$

This is consistent with the result in the previous section if $\lambda_{0} \sim a^{-1}$.

\subsection{Relation to expansion around Schwarzschild metric}

In the previous section, we have calculated the perturbative expansion around the Schwarzschild solution assuming that $r-a \sim \mathcal{O}\left(\kappa^{0}\right)$, and shown that the expression in terms of the proper radial coordinate $z$ gives a good expansion even for $r-a \sim \mathcal{O}(\kappa)$. The second order corrections do not contribute to the leading order terms, which is of $\mathcal{O}(\kappa)$ of the perturbative expansion for $r-a \sim \mathcal{O}(\kappa)$, and it is expected that the higher order terms of the expansion for $r-a \sim \mathcal{O}\left(\kappa^{0}\right)$ would not affect the leading order terms of the expansion for $r-a \sim \mathcal{O}(\kappa)$.

The perturbative expansion for $r-a \sim \mathcal{O}\left(\kappa^{0}\right)$ is expressed in terms of $z$ as eqs. (4.21) and (4.22). The expansions of $\rho$ and $r$ are

$$
\begin{aligned}
& \rho(z)=\log \left(\lambda^{2} a^{2}\right)+\log \left(\frac{z^{2}}{4 a^{2}}\right)-\frac{z^{2}}{3 a^{2}}+\cdots, \\
& r(z)=a+\frac{z^{2}}{4 a}+2 a \kappa q \log \left(\frac{z}{a}\right)+\cdots .
\end{aligned}
$$

Here, we have introduced an additional constant $\lambda$ which can be absorbed by a redefinition of the time coordinate $t$. The proper radial coordinate $z$ is related to the tortoise coordinate $x$ by (5.3), and the relation can be expressed as

$$
x-x_{0} \simeq \frac{2}{\lambda} \log \left(\frac{z}{a}\right)+\frac{z^{2}}{6 a^{2} \lambda} .
$$

For small $z$ as $z^{2} \sim \mathcal{O}(\kappa)$, the constant $x_{0}$ should behave as $x_{0} \sim \log \kappa$, and hence we write

$$
x_{0} \equiv-\frac{1}{2 \lambda} \log \left(4 \kappa \hat{c}_{0}\right) \text {. }
$$

Then, $z$ can be expressed as

$$
\frac{z}{a}=2\left(\kappa \hat{c}_{0}\right)^{1 / 2} e^{\lambda x / 2}\left(1-\frac{\kappa \hat{c}_{0}}{3} e^{\lambda x}\right)
$$

As a result, $\rho$ and $r$ are rewritten as

$$
\begin{aligned}
e^{\rho(x)} & =\kappa \hat{c}_{0} e^{\lambda x}\left(1-2 \kappa \hat{c}_{0} e^{\lambda x}\right)+\mathcal{O}\left(\kappa^{3}\right), \\
r(x) & =a+a \kappa q \log \left(4 \kappa \hat{c}_{0}\right)+\kappa \hat{c}_{0} e^{\lambda x}+a \kappa q \lambda x+\mathcal{O}\left(\kappa^{2}\right) .
\end{aligned}
$$

Here, the additional constant $a \kappa q \log \left(4 \kappa \hat{c}_{0}\right)$ can be absorbed by a redefinition of $a$. 
The expressions above agree with (5.18) and (5.19) if

$$
\begin{aligned}
\hat{c}_{0} & =\tilde{c}_{0}, \\
\lambda & =\lambda_{0}=\frac{1}{a} .
\end{aligned}
$$

This implies that the perturbative expansions for $z^{2} \sim \mathcal{O}\left(\kappa^{0}\right)$ (eqs. (4.21) and (4.22)) completely reproduce the leading order terms of the perturbative expansions for $z^{2} \sim \mathcal{O}(\kappa)$, and hence the higher order corrections to eqs. (4.21) and (4.22) do not contribute to the leading order terms of the perturbative expansion around the neck. The constant $\lambda$ is fixed here since a boundary condition is imposed on the expansion around the Schwarzschild metric.

\section{Non-perturbative analysis}

In the perturbative analysis, we have seen that the near-horizon region can be classified into the following three categories: (1) wormhole-like throat: there is a local minimum of the areal radius, resembling the throat of a traversable wormhole, (2) black-hole horizon: it is essentially the Schwarzschild event horizon. (3) neither of the above. In this section, we shall find non-perturbative solutions to the semi-classical Einstein equations. They are also classified into three categories that are compatible with the perturbative result, while the non-perturbative solutions also demonstrate new features that are not shown in the perturbative analysis.

The metric that we will use for the non-perturbative analysis is eq. (2.7), which describes a generic static, spherically symmetric geometry. For the reader's convenience, it is repeated here:

$$
d s^{2}=-\frac{e^{A(z)}}{B^{2}(z)} d t^{2}+d z^{2}+B(z) d \Omega^{2} .
$$

We shall solve the functions $A(z)$ and $B(z)$ in a neighborhood of an arbitrary point from the semi-classical Einstein equation.

As we wish to consider the most general vacuum energy-momentum tensor for a conformal matter field, the only constraints on the vacuum energy-momentum tensor are (i) the anomaly condition (2.2) and (ii) the conservation law (2.10). Similar to the perturbative analysis in section (4), our strategy is to first write down the most general conserved energy-momentum tensor $\left\langle T^{\mu}{ }_{\nu}\right\rangle$ compatible with these two constraints, and then solve two independent semi-classical Einstein equations, e.g.

$$
G_{z}^{z}=\kappa\left\langle T_{z}^{z}\right\rangle \quad \text { and } \quad G^{\mu}{ }_{\mu}=\kappa\left\langle T_{\mu}^{\mu}\right\rangle .
$$

While the trace $\left\langle T^{\mu}{ }_{\mu}\right\rangle$ is fixed by the anomaly (2.2), the energy-momentum is now uniquely determined by $\left\langle T^{z}{ }_{z}\right\rangle$. Equivalently, it is fixed by a constant parameter $q$ together with a functional degree of freedom that can be attributed to $\Theta$ or $\left\langle T^{\theta}{ }_{\theta}\right\rangle$, as in (2.36) with (2.41) and (2.42).

With the generic conserved energy-momentum tensor given above, we only need to solve two of the semi-classical equations (6.2), which are

$$
G_{z}^{z}=\kappa\left(B(z) e^{-A(z)} q+f(z)\right)
$$


and

$$
G^{\mu}{ }_{\mu}=\kappa\left(c_{4} \mathcal{F}+a_{4} \mathcal{G}\right),
$$

where $f(z)$ is different from (3.13) but defined by

$$
f(z) \equiv B(z) e^{-A(z)}[H(z)+G(z)],
$$

and $H(z)$ and $G(z)$ are given by (2.41) and (2.42).

By integrating (6.3), $A(z)$ is expressed in term of $B(z)$ as

$$
A(z)=A_{0}+\int_{z_{0}}^{z} K\left(z^{\prime}\right) d z^{\prime}+\log \left\{1+2 \kappa q \int_{z_{0}}^{z} \exp \left[-A_{0}-\int_{z_{0}}^{z^{\prime}} K\left(z^{\prime \prime}\right) d z^{\prime \prime}\right] \frac{B^{2}\left(z^{\prime}\right)}{B^{\prime}\left(z^{\prime}\right)} d z^{\prime}\right\},
$$

where

$$
K(z)=\frac{2}{B^{\prime}(z)}(1+\kappa f(z) B(z))+\frac{3 B^{\prime}(z)}{2 B(z)},
$$

and $A_{0}$ is the integration constant. Then eq. (6.4) gives the differential equation for $B(z)$. It is hard to find exact solutions to (6.4), so we shall solve them in a small neighborhood around a fixed point $z=z_{0}$. In eq. (6.6), we have used an integral from $z_{0}$ to $z$, but it can be replaced by a different starting point for integration with an appropriate value of $A_{0}$ In general, integrands may diverge at $z=z_{0}$, and then the integration would be defined such that it has no constant part in its expansion around $z=z_{0}$.

Since we do not intend to attack the UV problem close to the origin, we shall always assume that the areal radius $r$ is large in this neighborhood, so that the function $B(z)$ (which is $r^{2}$ ) is finite and much larger than $\kappa$. On the other hand, the function $A(z)$ can be either finite or diverging. $\left(A(z) \rightarrow-\infty\right.$ as $z \rightarrow z_{0}$ if there is a horizon at $z_{0}$.)

\subsection{Solving $A(z)$ and $B(z)$}

In a sufficiently small neighborhood of a generic point $z=z_{0}$ which is not a horizon, we can expand the functions $A(z)$ and $B(z)$ in power of $z$ as

$$
\begin{aligned}
& A(z)=A_{0}+A_{1}\left(z-z_{0}\right)+A_{2}\left(z-z_{0}\right)^{2}+A_{3}\left(z-z_{0}\right)^{3}+\cdots, \\
& B(z)=B_{0}+B_{1}\left(z-z_{0}\right)+B_{2}\left(z-z_{0}\right)^{2}+B_{3}\left(z-z_{0}\right)^{3}+\cdots .
\end{aligned}
$$

Plugging them into the semi-classical Einstein equations (6.3) and (6.4), we find straightforwardly the solutions for the coefficients $A_{n}, B_{n}$ :

$$
A_{1}=\frac{2}{B_{1}}+\frac{3 B_{1}}{2 B_{0}}+\frac{2 \kappa B_{0}}{B_{1}}\left\langle T_{z}^{z}\left(z_{0}\right)\right\rangle
$$

where $\left\langle T_{z}^{z}\left(z_{0}\right)\right\rangle=\left[q_{z_{0}} B_{0} e^{-A_{0}}+f\left(z_{0}\right)\right]$, and

$$
\begin{aligned}
& A_{2}=-\frac{B_{0}^{2} A_{1}^{2}+3 B_{1}^{2}-2 B_{0}\left(2+A_{1} B_{1}\right)}{4 B_{0}^{2}}+\mathcal{O}(\kappa), \\
& B_{2}=\frac{B_{1}\left(-8 B_{0}+B_{0}^{2} A_{1}^{2}-3 B_{1}^{2}\right)}{4 B_{0}\left(B_{0} A_{1}-3 B_{1}\right)}+\mathcal{O}(\kappa),
\end{aligned}
$$




$$
\begin{aligned}
& A_{3}=\frac{64 B_{0}^{3}+16 B_{0}^{2} B_{1}^{2}-52 B_{0} B_{1}^{4}+27 B_{1}^{6}}{96 B_{0}^{3} B_{1}^{3}}+\mathcal{O}(\kappa), \\
& B_{3}=\frac{4 B_{0} B_{1}-B_{1}^{3}}{48 B_{0}^{2}}+\mathcal{O}(\kappa),
\end{aligned}
$$

etc. for arbitrary non-zero constants $B_{0}, B_{1}$.

Notice that this solution is not unique. There is in fact another class of solutions given by eq. (6.10) and

$$
\begin{aligned}
& A_{2}=\frac{3\left(B_{0} A_{1}-3 B_{1}\right)^{2}}{2 \kappa c_{4} B_{0}^{2} A_{1}^{2}}+\mathcal{O}\left(\kappa^{0}\right), \\
& B_{2}=-\frac{3\left(B_{0} A_{1}-3 B_{1}\right) B_{1}}{2 \kappa c_{4} B_{0} A_{1}^{2}}+\mathcal{O}\left(\kappa^{0}\right), \\
& A_{3}=\frac{288 B_{0}^{2} B_{1}^{3}\left(4 B_{0}-3 B_{1}^{2}\right)^{2}}{\kappa^{2} c_{4}^{2}\left(4 B_{0}+3 B_{1}^{2}\right)^{5}}+\mathcal{O}\left(\kappa^{-1}\right), \\
& B_{3}=\frac{48 B_{0}^{3} B_{1}^{3}\left(4 B_{0}-9 B_{1}^{2}\right)\left(4 B_{0}-3 B_{1}^{2}\right)}{\kappa^{2} c_{4}^{2}\left(4 B_{0}+3 B_{1}^{2}\right)^{5}}+\mathcal{O}\left(\kappa^{-1}\right),
\end{aligned}
$$

etc. This solution has a singular limit as $\kappa \rightarrow 0$. The energy-momentum tenor for this configuration is of the Planck scale, presumably due to the presence of classical conformal matter, rather than being that of a vacuum state. According to the Buchdahl theorem [33], any static classical matter configuration with a radius less than $9 / 8$ of the Schwarzschild radius must suffer divergence in pressure. What we see here is that a regular solution exists for conformal matter due to quantum effect. The energy density and pressure are regularized to the Planck scale. The same effect was shown in ref. [22] with the $2 \mathrm{D}$ model for the energy-momentum tensor. It was also obtained in a 4D model [26-32].

We shall focus on the solution (6.10)-(6.14) in the following.

Notice also that eq. (6.10) is singular if either $B_{0}$ or $B_{1}$ vanishes. While $B_{0} \gg \kappa$ as it is the areal radius squared at $z=z_{0}$, it is possible that $B_{1}$ vanishes at $z=z_{0}$, which means that it is a local minimum of the areal radius.

For the case $B_{1} \neq 0, A_{0}$ is arbitrary, and all other coefficents are fixed for given $A_{0}, B_{0}$ and $B_{1}$ as in eqs. (6.10)-(6.14). For the solutions in which there is neither a horizon nor a wormhole-like structure, the geometry is given by this solution everywhere in in vacuum as long as $B_{0}$ is sufficiently large.

On the other hand, even if $B_{1} \neq 0$ at $z=z_{0}$, there may be another point $z=z_{0}^{\prime}$ where $B_{1}$ vanishes when $B(z)$ is expanded around $z_{0}^{\prime}$.

There are thus three classes of solutions. One class of solutions has $A(z)$ diverging at a certain point $z=z_{0}$, to be discussed in section 6.3. For the other two classes, $A(z)$ is finite everywhere, but $B(z)$ either has or has no local minimum. We will focus on the class of solutions with a local minimum of $B(z)$ in section 6.2 .

Regardless of whether $B_{1}$ vanishes, the tangential pressure at the point $z=z_{0}$ is $\left\langle T_{\theta \theta}(0)\right\rangle=\mathcal{O}(\kappa)$ for the solution (6.10)-(6.14). (Incidentally, $\left\langle T_{\theta \theta}(0)\right\rangle=\mathcal{O}\left(\kappa^{-1}\right)$ for the solution eqs. (6.15)-(6.18).) 


\subsection{Wormhole-like throat $(q<0)$}

In this subsection, we focus on the nonperturbative solution to the semi-classical Einstein equations that has a wormhole-like structure, that is, there is a local minimum of the areal radius $r$, and we study the solution in a small neighborhood of the wormhole neck.

If $r=a$ is located in finite proper distance from finite $r$ with $r \neq a, B(z)$ should have a regular expansion around $r=a$, as (6.9):

$$
B(z)=a^{2}+B_{1}\left(z-z_{0}\right)+B_{2}\left(z-z_{0}\right)^{2}+B_{3}\left(z-z_{0}\right)^{3}+\cdots .
$$

Let us assume that $B_{1}=0$, then $z=z_{0}$ is the local minimum of $r$, and $B(z)$ is expanded as

$$
B(z)=a^{2}+B_{2}\left(z-z_{0}\right)^{2}+B_{3}\left(z-z_{0}\right)^{3}+\cdots,
$$

with $B_{2}$ positive. ${ }^{5}$ Here, $a$ is nothing but the areal radius of the wormhole neck, to which we shall refer as the quantum Schwarzschild radius. Then $K(z)$, defined by eq. (6.7), behaves as

$$
K(z)=\frac{1+a^{2} \kappa f\left(z_{0}\right)}{B_{2}} \frac{1}{z}+(\text { finite }),
$$

and hence, the second term in (6.6) has a logarithmic divergence in the limit $z \rightarrow z_{0}$. For $q \neq 0$, the third term in (6.6) behaves as

$$
\log \left[-e^{-A_{0}} \frac{\kappa q a^{4}}{1+a^{2} \kappa f\left(z_{0}\right)} z^{-\left(1+a^{2} f\left(z_{0}\right)\right) / B_{2}}+\cdots\right]
$$

and hence, the logarithmic divergences in the second and third terms in (6.6) cancel. The integration constant $A_{0}$ in the expression above is also cancelled with the first term in (6.6), and $A(z)$ is then expanded as

$$
A(z)=\log \left[-\frac{\kappa q a^{4}}{1+a^{2} \kappa f\left(z_{0}\right)}\right]+\cdots,
$$

in which the leading order term is real only if $q<0$. This implies that the assumption (6.20), or equivalently, the local minimum of $r$ appears only if $q<0$. This is consistent with the results in the previous sections.

Now, we have seen that $A(z)$ and $B(z)$ do not diverge for $q<0$ and have regular expansion as (6.8) and (6.9). Next, we consider (6.8) and (6.9) in more details and calculate the coefficient. Assuming that the local minimum is located at $z=z_{0}$, so the expansion of $B(z)(6.9)$ around this point has $B_{1}=0$. Then eq. (6.10) implies that $A_{1}$ remains arbitrary, but $A_{0}$ and $B_{0}$ must satisfy the relation

$$
\left\langle T_{z}^{z}\left(z_{0}\right)\right\rangle=q_{z_{0}} B_{0} e^{-A_{0}}+f\left(z_{0}\right)=-\frac{1}{\kappa B_{0}} .
$$

\footnotetext{
${ }^{5}$ To be more precise, $B_{2}$ can be negative in general, but $B_{2}$ must be non-negative at the first zero of $B_{1}$ as one moves towards the center from distance, since $B(z)$ is an increasing function in the asymptotic region. The argument here (that $A(z)$ does not diverge) can be generalized to $B_{2}=0$ with $B_{n}>0$ for the first non-zero $B_{n}$ with $n>0$.
} 
All other coefficients are fixed for given $A_{0}$ (or $B_{0}$ ) and $A_{1}$. There are only 2 free parameters, instead of 3 as the case $B_{1} \neq 0$ simply because the location $z_{0}$ of the local minimum of $B(z)$ is another free parameter.

Let us go through the derivation of the solutions (6.10)-(6.14) and (6.15)-(6.18) for the special case of the wormhole-like structure in more detail. First, while $A_{1}$ is a free parameter, $A_{2}$ and $B_{2}$ are solved from the semi-classical Einstein equation as

$$
\begin{aligned}
& A_{2}=Y-\frac{A_{1}^{2}}{4}, \\
& B_{2}=a^{2} Y-\kappa\left\langle T_{\theta \theta}\left(z_{0}\right)\right\rangle,
\end{aligned}
$$

where

$$
\begin{aligned}
Y \equiv & \left(16 \kappa a^{4} c_{4}\right)^{-1}\left\{3 a^{4}+4 a^{2} \kappa\left[c_{4}\left(6 \kappa\left\langle T_{\theta \theta}\left(z_{0}\right)\right\rangle-2\right)-3 a_{4}\right]\right. \\
& \left. \pm \sqrt{9 a^{8}+72 \kappa a^{6}\left(2 \kappa c_{4}\left\langle T_{\theta \theta}\left(z_{0}\right)\right\rangle-2 c_{4}-a_{4}\right)+48 a^{4} a_{4} \kappa^{2}\left(4 \kappa c_{4}\left\langle T_{\theta \theta}\left(z_{0}\right)\right\rangle+4 c_{4}+3 a_{4}\right)}\right\}
\end{aligned}
$$

The parameter $Y$ has two solutions for the different choices of the sign in eq. (6.27). In the $\kappa$-expansion, the solution with the - sign is approximately

$$
Y=\frac{1}{a^{2}}+\kappa \frac{6 c_{4}+4 a_{4}}{a^{4}}+\mathcal{O}\left(\kappa^{2}\right)
$$

The solution with the + sign is approximately

$$
Y=\frac{3}{8 \kappa c_{4}}-\frac{4 c_{4}+3 a_{4}}{2 a^{2} c_{4}}+\mathcal{O}\left(\kappa^{2}\right),
$$

which blows up in the limit $\kappa \rightarrow 0$. This means that the energy-momentum tensor is not that of the vacuum of the conformal field. The same comments below eq. (6.18) apply here.

Demanding that the solution has a classical limit, we should take the first solution (6.28) of $Y$. Then

$$
B_{2}=1+\kappa \frac{6 c_{4}+4 a_{4}}{a^{2}}-\kappa\left\langle T_{\theta \theta}\left(z_{0}\right)\right\rangle+\mathcal{O}\left(\kappa^{2}\right) .
$$

When the conformal charges $c_{4}, a_{4}$ vanish, in the absence of the pressure $\left\langle T_{\theta \theta}\left(z_{0}\right)\right\rangle$, this expression reproduces the result $B_{2}=1$ of the Schwarzschild solution. (But there is no neck in the Schwarzschild solution at $z=z_{0}$ because it coincides with the horizon.) For a large black hole, since $\kappa$ is very small, $B_{2}$ is positive unless $\left\langle T_{\theta \theta}\left(z_{0}\right)\right\rangle \gtrsim \mathcal{O}\left(\kappa^{-1}\right)$, and there is a local minimum of the areal radius at $z=z_{0}$.

Next we can solve the coefficients $A_{3}$ and $B_{3}$. They are

$$
\begin{aligned}
A_{3}= & -2\left[3 a^{2}\left(-3 a^{2}+8 \kappa c_{4}+16 \kappa a^{2} c_{4} Y-24 \kappa^{2} c_{4}\left\langle T_{\theta \theta}\left(z_{0}\right)\right\rangle+12 \kappa a_{4}\right)\right]^{-1} \\
& {\left[\frac{3}{8} a^{4} A_{1}^{3}-2 \kappa a^{4} c_{4} A_{1}^{3} Y+6 \kappa a^{4} a_{4} A_{1} Y^{2}-\kappa a^{2} c_{4} A_{1}^{3}-\frac{3}{2} \kappa a^{2} a_{4} A_{1}^{3}-\frac{3}{2} \kappa a^{2} A_{1}\left\langle T_{\theta \theta}\left(z_{0}\right)\right\rangle\right.} \\
& +3 \kappa^{2} a^{2} c_{4} A_{1}^{3}\left\langle T_{\theta \theta}\left(z_{0}\right)\right\rangle+8 \kappa^{2} a^{2} c_{4} A_{1}\left\langle T_{\theta \theta}\left(z_{0}\right)\right\rangle Y-12 \kappa^{2} a^{2} a_{4} A_{1}\left\langle T_{\theta \theta}\left(z_{0}\right)\right\rangle Y
\end{aligned}
$$




$$
\begin{aligned}
& +4 \kappa^{2} c_{4} A_{1}\left\langle T_{\theta \theta}\left(z_{0}\right)\right\rangle-6 \kappa^{2} c_{4}\left\langle T_{\theta \theta}^{\prime}\left(z_{0}\right)\right\rangle+6 \kappa^{2} a_{4} A_{1}\left\langle T_{\theta \theta}\left(z_{0}\right)\right\rangle-12 \kappa^{2} a_{4}\left\langle T_{\theta \theta}^{\prime}\left(z_{0}\right)\right\rangle \\
& -12 \kappa^{2} a^{2} c_{4} Y\left\langle T_{\theta \theta}^{\prime}\left(z_{0}\right)\right\rangle-12 \kappa^{3} c_{4} A_{1}\left\langle T_{\theta \theta}\left(z_{0}\right)\right\rangle^{2}+6 \kappa^{3} a_{4} A_{1}\left\langle T_{\theta \theta}\left(z_{0}\right)\right\rangle^{2} \\
& \left.+18 \kappa^{3} c_{4}\left\langle T_{\theta \theta}\left(z_{0}\right)\right\rangle\left\langle T_{\theta \theta}^{\prime}\left(z_{0}\right)\right\rangle\right] \\
\simeq & \frac{1}{12} A_{1}^{3}+\frac{\kappa}{24} A_{1}\left[32 a_{4} Y^{2}-\frac{\left\langle T_{\theta \theta}\left(z_{0}\right)\right\rangle}{a^{2}}\right]+\mathcal{O}\left(\kappa^{2}\right), \\
B_{3}= & -\left[3\left(-3 a^{2}+\kappa\left(8 c_{4}+12 a_{4}+16 a^{2} c_{4} Y\right)-24 \kappa^{2} c_{4}\left\langle T_{\theta \theta}\left(z_{0}\right)\right\rangle\right)\right]^{-1} \\
& {\left[-\frac{3}{2} a^{4} A_{1} Y+8 \kappa a^{4} c_{4} A_{1} Y^{2}+12 \kappa a^{4} a_{4} A_{1} Y^{2}+4 \kappa a^{2} c_{4} A_{1} Y+\frac{3}{2} \kappa a^{2} A_{1}\left\langle T_{\theta \theta}\left(z_{0}\right)\right\rangle\right.} \\
& -3 \kappa a^{2}\left\langle T_{\theta \theta}^{\prime}\left(z_{0}\right)\right\rangle+6 \kappa a^{2} a_{4} A_{1} Y-24 \kappa^{2} a^{2} a_{4} A_{1}\left\langle T_{\theta \theta}\left(z_{0}\right)\right\rangle Y \\
& -20 \kappa^{2} a^{2} c_{4} A_{1}\left\langle T_{\theta \theta}\left(z_{0}\right)\right\rangle Y-4 \kappa^{2} c_{4}\left\langle T_{\theta \theta}^{\prime}\left(z_{0}\right)\right\rangle-8 \kappa^{2} a^{2} c_{4} Y\left\langle T_{\theta \theta}^{\prime}\left(z_{0}\right)\right\rangle \\
& -6 \kappa^{2} a_{4} A_{1}\left\langle T_{\theta \theta}\left(z_{0}\right)\right\rangle-12 \kappa^{2} a_{4}\left\langle T_{\theta \theta}^{\prime}\left(z_{0}\right)\right\rangle+\kappa^{3} 12 c_{4} A_{1}\left\langle T_{\theta \theta}\left(z_{0}\right)\right\rangle^{2} \\
& \left.-4 \kappa^{2} c_{4} A_{1}\left\langle T_{\theta \theta}\left(z_{0}\right)\right\rangle+12 \kappa^{3} c_{4}\left\langle T_{\theta \theta}\left(z_{0}\right)\right\rangle\left\langle T_{\theta \theta}^{\prime}\left(z_{0}\right)\right\rangle+12 \kappa^{3} a_{4} A_{1}\left\langle T_{\theta \theta}\left(z_{0}\right)\right\rangle^{2}\right] \\
\simeq & -\frac{A_{1}}{6}-\frac{\kappa}{3}\left[A_{1}\left(3 c_{4}-2 a_{4}\right)-\frac{1}{2} A_{1}\left\langle T_{\theta \theta}\left(z_{0}\right)\right\rangle+\left\langle T_{\theta \theta}^{\prime}\left(z_{0}\right)\right\rangle\right]+\mathcal{O}\left(\kappa^{2}\right) .
\end{aligned}
$$

In the last line of the expressions of $A_{3}$ and $B_{3}$, we have used the value of $Y$ in eq. (6.28).

Plugging these back into $A(z)$ and $B(z)$, we get

$$
\begin{aligned}
A(z)= & A_{0}+A_{1} z+\left(\frac{1}{a^{2}}-\frac{1}{4} A_{1}^{2}\right) z^{2}+\frac{A_{1}^{3}}{12} z^{3}+\mathcal{O}\left(z^{4}\right) \\
& +\kappa\left[\frac{2\left(3 c_{4}+2 a_{4}\right)}{a^{4}} z^{2}+\frac{A_{1}\left(4 a_{4}-a^{2}\left\langle T_{\theta \theta}\left(z_{0}\right)\right\rangle\right)}{3 a^{4}} z^{3}+\mathcal{O}\left(z^{4}\right)\right]+\mathcal{O}\left(\kappa^{2}\right), \\
B(z)= & a^{2}+z^{2}-\frac{A_{1}}{6} z^{3}+\mathcal{O}\left(z^{4}\right) \\
& +\kappa\left[\frac{2\left(3 c_{4}+2 a_{4}\right)}{a^{2}} z^{2}-\frac{2\left(3 c_{4}-2 a_{4}\right) A_{1}-A_{1}\left\langle T_{\theta \theta}\left(z_{0}\right)\right\rangle+2\left\langle T_{\theta \theta}^{\prime}\left(z_{0}\right)\right\rangle}{6} z^{3}+\mathcal{O}\left(z^{4}\right)\right] \\
& +\mathcal{O}\left(\kappa^{2}\right) .
\end{aligned}
$$

For given Weyl anomaly (given $c_{4}$ and $a_{4}$ ) and given tangential pressure at the neck $\left\langle T_{\theta \theta}\left(z_{0}\right)\right\rangle$, these solutions are parametrized by 3 parameters: $a, A_{1}$ and $z_{0}$. However, this solution around the neck should be continuously connected to the asymptotic Schwarzschild solution at large distances. We expect that once the classical Schwarzschild radius $a_{0}$ is fixed, only two parametric constants remain independent, corresponding to the freedom in specifying the parameter $q_{z_{0}}$ and the tangential pressure at the neck $\left\langle T_{\theta \theta}\left(z_{0}\right)\right\rangle$.

\subsection{Event horizon $(q=0)$}

In this subsection, we consider the class of solutions with even horizons. For $q=0$, the third term in (6.6) vanishes. As is discussed in section 6.2, for the expansion (6.20), $A(z)$ 
has the logarithmic divergence at $z=z_{0}$ and is expanded as

$$
A(z)=A_{0}+\frac{1+a^{2} \kappa f\left(z_{0}\right)}{B_{2}} \frac{1}{z-z_{0}}+\cdots .
$$

This implies that there is the event horizon at $z=z_{0}$. The coefficients in the expansion of $B(z)$ are determined by (6.4) and calculated, for example, as

$$
\begin{aligned}
& B_{2}=\frac{1}{2}\left(1+a^{2} \kappa f\left(z_{0}\right)\right), \\
& B_{3}=\frac{a^{2}}{3} f^{\prime}\left(z_{0}\right) .
\end{aligned}
$$

Hence $A(z)$ and $B(z)$ are expanded as

$$
\begin{aligned}
& A(z)=A_{0}+2 \log \left(z-z_{0}\right)+\mathcal{O}\left(\left(z-z_{0}\right)^{2}\right) \\
& B(z)=a^{2}+\frac{1}{2}\left(1+a^{2} \kappa f\left(z_{0}\right)\right)\left(z-z_{0}\right)^{2}+\mathcal{O}\left(\left(z-z_{0}\right)^{3}\right) .
\end{aligned}
$$

This is consistent with the results (4.16) and (5.24) in the previous sections.

In a small neighborhood of the horizon, we take the ansatz

$$
\begin{aligned}
& A(z)=A_{0}+2 d_{0} \log \left(z+d_{1} z^{2}+d_{2} z^{3}+d_{3} z^{4}+\cdots\right), \\
& B(z)=a^{2}+B_{1} z+B_{2} z^{2}+B_{3} z^{3}+B_{4} z^{4}+\cdots,
\end{aligned}
$$

where we have shifted the $z$-coordinate such that the horizon is located at $z=0$ and we can also scale the $t$-coordinate to set $A_{0}=0$.

The semi-classical Einstein equations (6.3), (6.4) are solved order by order in the $z$ expansion. We find

$$
\begin{aligned}
d_{0}= & 1, \\
B_{1}= & 0 \\
d_{1}= & 0, \\
B_{2}= & \frac{a^{2}}{8 \kappa\left(4 c_{4}+3 a_{4}\right)}\left[3-\frac{4 \kappa\left(2 c_{4}+3 a_{4}\right)}{a^{2}}+\frac{8 \kappa^{2} c_{4}\left\langle T_{\theta \theta}(0)\right\rangle}{a^{2}}\right. \\
& \pm \frac{\sqrt{3}}{a^{2}}\left(3 a^{4}-48 \kappa a^{2}\left(c_{4}+a_{4}\right)+48 \kappa^{2}\left(\left(c_{4}+a_{4}\right) a_{4}+a^{2} c_{4}\left\langle T_{\theta \theta}(0)\right\rangle\right)\right. \\
& \left.\left.+24 \kappa^{2} a^{2} a_{4}\left\langle T_{\theta \theta}(0)\right\rangle+96 \kappa^{3}\left(c_{4}+a_{4}\right) c\left\langle T_{\theta \theta}(0)\right\rangle-16 \kappa^{4} c_{4} a_{4}\left\langle T_{\theta \theta}(0)\right\rangle^{2}\right)^{1 / 2}\right], \\
d_{2}= & \frac{4 B_{2}+\kappa\left\langle T_{\theta \theta}(0)\right\rangle}{6 a^{2}} .
\end{aligned}
$$

The choice of the plus sign in the solution of $B_{2}$ gives

$$
B_{2}=\frac{1}{2}+\kappa\left(\frac{3\left(c_{4}+a_{4}\right)}{a^{2}}-\frac{\left\langle T_{\theta \theta}\right\rangle}{2}\right)+\mathcal{O}\left(\kappa^{2}\right) .
$$

The choice of the minus sign gives a result

$$
B_{2}=\frac{3 a^{2}}{4 \kappa\left(4 c_{4}+3 a_{4}\right)}-\frac{8 c_{4}+9 a_{4}}{8 c_{4}+6 a_{4}}+\mathcal{O}(\kappa)
$$


that diverges as $\kappa \rightarrow 0$. The latter case clearly does not correspond to a vacuum state. The same comments below eq. (6.18) apply here.

With $B_{2}$ given by the solution with a finite value in the limit $\kappa \rightarrow \infty$, we find

$$
\begin{aligned}
B_{3} & =-\frac{2 \kappa}{9}\left\langle T_{\theta \theta}^{\prime}(0)\right\rangle+\mathcal{O}\left(\kappa^{2}\right), \\
d_{3} & =-\frac{\kappa}{18 a^{2}}\left\langle T_{\theta \theta}^{\prime}(0)\right\rangle+\mathcal{O}\left(\kappa^{2}\right) .
\end{aligned}
$$

Since $B_{1}=0$ and $B_{2}>0$, the function $B(z)$ also has a local extremum at $z=0$. But since $e^{A(z)}$ vanishes at $z=0$, it is a horizon and the solution does not apply to the range $z<0$. Hence it is in fact not really a local minimum.

For a given Weyl anomaly (given $c_{4}$ and $a_{4}$ ) and a given $\left\langle T_{\theta \theta}(0)\right\rangle$, these solutions are parametrized by the quantum Schwarzschild radius $a$ and the location of the horizon. When the solution is identified as part of a global solution which asymptotes to the Schwarzschild solution at large distances with a given classical Schwarzschild radius, there is only a single independent parameter, corresponding to the choice of the tangential pressure $\left\langle T_{\theta \theta}(0)\right\rangle$.

This is compatible with the perturbative analysis, where it has been shown that the existence of the horizon demands a fine-tuning of a parameter $q_{z_{0}}$ to $q_{z_{0}}=0$ exactly. Hence the number of parameters for the solutions with a horizon is one fewer than the other two classes of solutions.

\subsection{No neck \& no horizon $(q>0)$}

As we discussed in section 6.2, the expansion (6.20) for $q>0$ gives complex $A(z)$ at $z=z_{0}$. Since $A(z)$ must be real, this implies that $r$ has no local minimum in this case. Hence $B_{1}$ must be non-zero for $q>0$, and $K(z)$ and $A(z)$ have regular expansions only with positive powers of $z$ and thus there is no horizon.

\section{Conclusion}

We find in this paper that the event horizon of the Schwarzschild solution can be removed by the back reaction of quantum energy. Depending on the quantum state, more precisely, depending on the parameter $q$ in $\left\langle T_{r}^{r}\right\rangle$ (3.12), the near-horizon region can resemble a wormhole throat for $q<0$, or it has a horizon for $q=0$, or it has neither a throat nor a horizon for $q>0$. Notice that the horizon persists only if the parameter $q$ is fine-tuned to exactly zero. The black hole is horizonless as long as $q$ is not exactly zero. For definiteness, we have focused on $4 \mathrm{D}$ conformal matter fields so that the trace $\left\langle T^{\mu}{ }_{\mu}\right\rangle$ is uniquely fixed. But it should be clear from our calculation that, for a wide class of models, one would reach the same conclusion about the necessity of fine-tuning for horizon.

It is interesting to ask whether the parameter $q$ is always driven to vanish for a generic gravitational collapse. In the conventional model of black holes, the answer is proposed to be affirmative. But it is merely a folklore in the absence of a thorough study of gravitational collapses. On the other hand, in view of the fuzzball scenario [6-10] or the firewall proposal [11-13], the answer could be negative. A support for the negative answer also comes from $2 \mathrm{D}$ models. For the 2D model of vacuum energy used in ref. [15], the wormhole-like 
structure is observed to appear in a generic gravitational collapse in both analytical [24] and numerical studies [23].

Although we have only focused on static configurations in this paper. The quantum vacuum state outside the collapsing matter for an astronomical black hole is expected to change very slowly over time. The static solution should serve as a good approximation of this time-dependent process. It will therefore be very interesting to see how different models of evaporating black holes are connected to different solutions in this paper. For instance, a 4D self-consistent model which considers both the formation and evaporation processes [26-31] was shown to be compatible with $4 \mathrm{D}$ conformal anomaly [32], so it can be viewed as approximate time-dependent solutions corresponding to some of the solutions in this paper. As it does not have a horizon nor a wormhole-like throat, but it has a Planck-scale tangential pressure, the model is most likely related to the class of solutions given in eqs. (6.10), (6.15)-(6.18).

\section{Acknowledgments}

We thank Chong-Sun Chu, Wen-Yu Wen, Chen-Pin Yeh, Shoichi Kawamoto, Po Ning and Philip Argyrus for discussions. P.M.H. and Y.M. are supported in part by the Ministry of Science and Technology, R.O.C. (project no. 104-2112-M-002-003-MY3) and by National Taiwan University (project no. 105R8700-2). H.K. is partially supported by Japan Society of Promotion of Science (JSPS), Grants-in-Aid for Scientific Research (KAKENHI) Grants No.16K05322. Y.Y. is partially supported by Japan Society of Promotion of Science (JSPS), Grants-in-Aid for Scientific Research (KAKENHI) Grants No. 18 K13550 and 17H01148. Y.Y. is also partially supported by RIKEN iTHEMS Program.

Open Access. This article is distributed under the terms of the Creative Commons Attribution License (CC-BY 4.0), which permits any use, distribution and reproduction in any medium, provided the original author(s) and source are credited.

\section{References}

[1] S.W. Hawking, Particle creation by black holes, Commun. Math. Phys. 43 (1975) 199 [Erratum ibid. 46 (1976) 206] [INSPIRE].

[2] S.W. Hawking, Breakdown of predictability in gravitational collapse, Phys. Rev. D 14 (1976) 2460 [INSPIRE].

[3] S.W. Hawking, The information paradox for black holes, arXiv:1509.01147 [INSPIRE].

[4] S.D. Mathur, The information paradox: a pedagogical introduction, Class. Quant. Grav. 26 (2009) 224001 [arXiv:0909.1038] [INSPIRE].

[5] D. Marolf, The black hole information problem: past, present and future, Rept. Prog. Phys. 80 (2017) 092001 [arXiv: 1703. 02143] [INSPIRE].

[6] O. Lunin and S.D. Mathur, AdS/CFT duality and the black hole information paradox, Nucl. Phys. B 623 (2002) 342 [hep-th/0109154] [INSPIRE]. 
[7] O. Lunin and S.D. Mathur, Statistical interpretation of Bekenstein entropy for systems with a stretched horizon, Phys. Rev. Lett. 88 (2002) 211303 [hep-th/0202072] [INSPIRE].

[8] S.D. Mathur, The fuzzball proposal for black holes: an elementary review, Fortsch. Phys. 53 (2005) 793 [hep-th/0502050] [inSPIRE].

[9] K. Skenderis and M. Taylor, The fuzzball proposal for black holes, Phys. Rept. 467 (2008) 117 [arXiv:0804.0552] [INSPIRE].

[10] S.D. Mathur, Resolving the black hole causality paradox, arXiv:1703.03042 [INSPIRE].

[11] A. Almheiri, D. Marolf, J. Polchinski and J. Sully, Black holes: complementarity or firewalls?, JHEP 02 (2013) 062 [arXiv: 1207.3123] [INSPIRE].

[12] S.L. Braunstein, S. Pirandola and K. Życzkowski, Better late than never: information retrieval from black holes, Phys. Rev. Lett. 110 (2013) 101301 [arXiv:0907.1190] [InSPIRE].

[13] S.L. Braunstein, S. Pirandola and K. Życzkowski, Better late than never: information retrieval from black holes, Phys. Rev. Lett. 110 (2013) 101301 [arXiv:0907.1190].

[14] S.M. Christensen and S.A. Fulling, Trace anomalies and the Hawking effect, Phys. Rev. D 15 (1977) 2088 [INSPIRE].

[15] P.C.W. Davies, S.A. Fulling and W.G. Unruh, Energy momentum tensor near an evaporating black hole, Phys. Rev. D 13 (1976) 2720 [InSPIRE].

[16] P.-M. Ho and Y. Matsuo, Static black holes with back reaction from vacuum energy, Class. Quant. Grav. 35 (2018) 065012 [arXiv: 1703.08662] [INSPIRE].

[17] A. Fabbri et al., Semiclassical zero-temperature corrections to Schwarzschild spacetime and holography, Phys. Rev. D 73 (2006) 104023 [hep-th/0512167] [INSPIRE].

[18] A. Fabbri et al., Static quantum corrections to the Schwarzschild spacetime, J. Phys. Conf. Ser. 33 (2006) 457 [hep-th/0512179] [INSPIRE].

[19] S.N. Solodukhin, Can black hole relax unitarily?, hep-th/0406130 [INSPIRE].

[20] S.N. Solodukhin, Restoring unitarity in BTZ black hole, Phys. Rev. D 71 (2005) 064006 [hep-th/0501053] [INSPIRE].

[21] T. Damour and S.N. Solodukhin, Wormholes as black hole foils, Phys. Rev. D 76 (2007) 024016 [arXiv: 0704.2667] [INSPIRE].

[22] P.-M. Ho and Y. Matsuo, Static black hole and vacuum energy: thin shell and incompressible fluid, JHEP 03 (2018) 096 [arXiv: 1710.10390] [INSPIRE].

[23] R. Parentani and T. Piran, The internal geometry of an evaporating black hole, Phys. Rev. Lett. 73 (1994) 2805 [hep-th/9405007] [INSPIRE].

[24] P.-M. Ho and Y. Matsuo, On the near-horizon geometry of an evaporating black hole, JHEP 07 (2018) 047 [arXiv: 1804.04821] [INSPIRE].

[25] C. Berthiere, D. Sarkar and S.N. Solodukhin, The fate of black hole horizons in semiclassical gravity, Phys. Lett. B 786 (2018) 21 [arXiv:1712.09914] [INSPIRE].

[26] H. Kawai, Y. Matsuo and Y. Yokokura, A self-consistent model of the black hole evaporation, Int. J. Mod. Phys. A 28 (2013) 1350050 [arXiv:1302.4733] [inSPIRE].

[27] H. Kawai and Y. Yokokura, Phenomenological description of the interior of the Schwarzschild black hole, Int. J. Mod. Phys. A 30 (2015) 1550091 [arXiv:1409. 5784] [inSPIRE]. 
[28] P.-M. Ho, Comment on self-consistent model of black hole formation and evaporation, JHEP 08 (2015) 096 [arXiv: 1505.02468] [INSPIRE].

[29] H. Kawai and Y. Yokokura, Interior of black holes and information recovery, Phys. Rev. D 93 (2016) 044011 [arXiv: 1509.08472] [INSPIRE].

[30] P.-M. Ho, The absence of horizon in black-hole formation, Nucl. Phys. B 909 (2016) 394 [arXiv: 1510.07157] [INSPIRE].

[31] P.-M. Ho, Asymptotic black holes, Class. Quant. Grav. 34 (2017) 085006 [arXiv: 1609.05775] [INSPIRE].

[32] H. Kawai and Y. Yokokura, A model of black hole evaporation and 4D Weyl anomaly, Universe 3 (2017) 51 [arXiv:1701.03455] [INSPIRE].

[33] H.A. Buchdahl, General relativistic fluid spheres, Phys. Rev. 116 (1959) 1027 [INSPIRE]. 